Algebraic $8 \mathcal{G}$ Geometric $\mathcal{T}$ opology

Volume 4 (2004) 1177-1210

Published: 15 December 2004

ATG

\title{
Categorification of the Kauffman bracket skein module of $I$-bundles over surfaces
}

\author{
Marta M. Asaeda \\ JÓZEF H. PRZYTYCKI \\ ADAM S. SIKORA
}

\begin{abstract}
Khovanov defined graded homology groups for links $L \subset \mathbb{R}^{3}$ and showed that their polynomial Euler characteristic is the Jones polynomial of $L$. Khovanov's construction does not extend in a straightforward way to links in $I$-bundles $M$ over surfaces $F \neq D^{2}$ (except for the homology with $\mathbb{Z} / 2$ coefficients only). Hence, the goal of this paper is to provide a nontrivial generalization of his method leading to homology invariants of links in $M$ with arbitrary rings of coefficients.

After proving the invariance of our homology groups under Reidemeister moves, we show that the polynomial Euler characteristics of our homology groups of $L$ determine the coefficients of $L$ in the standard basis of the skein module of $M$. Therefore, our homology groups provide a "categorification" of the Kauffman bracket skein module of $M$. Additionally, we prove a generalization of Viro's exact sequence for our homology groups. Finally, we show a duality theorem relating cohomology groups of any link $L$ to the homology groups of the mirror image of $L$.
\end{abstract}

AMS Classification 57M27; 57M25, 57R56

Keywords Khovanov homology, categorification, skein module, Kauffman bracket

\section{Introduction}

In his seminal work [K1, Khovanov constructed graded homology groups for links $L \subset \mathbb{R}^{3}$ and proved that their polynomial Euler characteristic is the Jones polynomial of $L$. (Throughout this paper we use Viro's (framed) normalization of Khovanov homology groups.) The crucial element in Khovanov's construction is the representation of a link by its diagram in $D^{2}$ unique up to Reidemeister moves. Despite the fact that links in all $I$-bundles over surfaces allow analogous diagrammatic representations, Khovanov's construction does not extend in a 
straightforward way to such links. In this paper we overcome this difficulty and define homology invariants of links in all orientable $I$-bundles $M$ over surfaces $F \neq \mathbb{R P}^{2}$.

We say that a simple closed loop $\gamma \subset F$ is bounding if it bounds either a disk or a Möbius band in $F$. Let $\mathcal{C}(F)$ be the set of all unoriented, unbounding, simple closed curves in $F$ considered up to homotopy. In Sections 35, we construct homology groups, $H_{i j s}(L)$, of links $L \subset M$ for any $i, j \in \mathbb{Z}$, and $s \in \mathbb{Z C}(F)$, and we prove (Section 6) that these homology groups are invariant under isotopies of $L$ in $M$.

In Section [7, we construct a generalization of Viro's exact sequence, VV, Section 3.3], which associates with any skein triple of link diagrams in $F$ a long exact sequence of their homology groups.

In Section 8, we prove that if $F$ is orientable then the polynomial Euler characteristics of our homology groups $H_{i j s}(L)$ determine the coefficients of $L$ in the standard basis of the Kauffman bracket skein module. Additionally, we prove an analogous result for links in twisted $I$-bundles over unorientable surfaces.

In Section 9, we introduce cohomology groups of links and prove a duality theorem relating them to homology groups of the mirror image of $L$. Finally, we discuss the dependence of our homology groups of links on the $I$-bundle structure of the ambient 3-manifold in Section 10.

The methods of this paper are applied in [APS] to define a new homology theory for tangles.

Acknowledgments The first author was sponsored in part by NSF grant DMS-0202613. The second author was partially sponsored by grant TMP25051958. The third author was sponsored in part by NSF grants DMS-0307078 and DMS-0111298.

\section{Skein modules of band links}

Let $M$ be an orientable $I$-bundle over a surface $F$. Consequently, $M$ is either $F \times I$ for an orientable $F$, or $M$ is the twisted $I$-bundle over an unorientable surface $F$. In the latter case we assume that $F \neq \mathbb{R P}^{2}$. A band knot $K$ in $M$ is either

- an embedding of an annulus into $M$ such that the projection of its core into $F$ is a preserving orientation loop in $F$; or 
- an embedding of a Möbius band into $M$ such that the projection of its core into $F$ is a reversing orientation curve in $F$.

A disjoint union of band knots is called a band link. Each band link in $M$ is represented by a diagram in $F$. (All link diagrams are considered with their blackboard framing. Hence, an orientation reversing loop in $F$ represents a Möbius band in $M$.) From now on, all links considered in this paper are band links, unless stated otherwise.

Denote the set of all band links in an orientable $I$-bundle $M$ over $F$, by $\mathcal{L}_{b}(M)$. For a given ring $R$ with a distinguished element $A^{ \pm 1}$, the skein module of band links in $M, \mathcal{S}_{b}(M ; R)$, is the quotient of $R \mathcal{L}_{b}(M)$ by the standard Kauffman bracket skein relations:

$$
\left.\searrow=A^{\smile}+A^{-1}\right)\left(, \quad L \cup \bigcirc=-\left(A^{2}+A^{-2}\right) L .\right.
$$

Although the definition of a band link $L \subset M$ depends on the $I$-bundle structure of $M$, the theorem below shows the skein module $\mathcal{S}_{b}(M ; R)$ (considered up to an isomorphism of $R$-modules) does not. Let $\mathcal{B}(F)$ (respectively: $\mathcal{B}_{n b}(F)$ ) be the set of all link diagrams in $F$ with no crossings and with no trivial (respectively: no bounding) components. Both $\mathcal{B}(F)$ and $\mathcal{B}_{n b}(F)$ contain the empty link, $\emptyset$. Following the proof of $[\mathrm{Pr}$, Theorem 3.1], one shows:

Theorem $2.1 \mathcal{S}_{b}(M ; R)$ is a free $R$-module with a basis composed by (band) links represented by diagrams in $\mathcal{B}(F)$.

Consequently, $\mathcal{S}_{b}\left(M ; \mathbb{Z}\left[A^{ \pm 1}\right]\right)=\mathcal{S}\left(M ; \mathbb{Z}\left[A^{ \pm 1}\right]\right)$ as $R$-modules despite the fact that there is no obvious explicit isomorphism between these modules for unorientable $F$. On the other hand, there is a natural isomorphism between $\mathcal{S}_{b}(M ; R)$ and $\mathcal{S}(M ; R)$ for any ring $R$ containing $\sqrt{-A}$. For such $R$, we have an isomorphism $\lambda: \mathcal{S}_{b}\left(M ; \mathbb{Z}\left[A^{ \pm 1}\right]\right) \rightarrow \mathcal{S}\left(M ; \mathbb{Z}\left[A^{ \pm 1}\right]\right)$ sending $L=K_{1} \cup \ldots \cup K_{n}$ to $(-A)^{3 k(L) / 2} K_{1}^{\prime} \cup \ldots \cup K_{n}^{\prime}$, where $K_{i}^{\prime}=K_{i}$ if $K_{i}$ is an annulus and, otherwise, $K_{i}^{\prime}$ is obtained from $K_{i}$ by adding a negative half-twist to $K_{i}$. Here $k(L)$ denotes the number of Möbius bands among the components, $K_{i}$, of $L$.

\section{Chain groups}

Let $D$ be a link diagram in $F$. Following $[\mathrm{V}$, a Kauffman state of $D$ is an assignment of +1 or -1 marker to each of the crossings of $D .1$ An enhanced

\footnotetext{
${ }^{1}$ J.B.Listing in $1847[\mathrm{Li}]$ was the first to consider two types of markers of a crossing: dexiotropic and laeotropic.
} 
(Kauffman) state of $D$ is a Kauffman state with an additional assignment of + or - sign to each closed loop obtained by smoothing the crossings of $D$ according to the following convention:

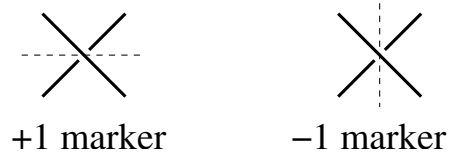

Such closed loops will be called by us "circles".

Denote the set of enhanced states of $D$ by $\mathcal{S}(D)$. The following notation is used throughout the paper for any $S \in \mathcal{S}(D)$ :

$$
\begin{aligned}
& I(S)=\sharp\{\text { positive markers }\}-\sharp\{\text { negative markers }\} . \\
& J(S)=I(S)+2 \tau(S), \text { where } \\
& \tau(S)=\sharp\{\text { positive trivial circles }\}-\sharp\{\text { negative trivial circles }\} .
\end{aligned}
$$

A circle is trivial if it bounds a disk in $F$. Furthermore, we say that a circle is bounding if it bounds either a disk or a Möbius band in $F$. Let $\mathcal{C}(F)$ be the set of all unoriented, unbounding, simple closed curves in $F$ considered up to homotopy. If the unbounding components of an enhanced state $S$ are $\gamma_{1}, \ldots, \gamma_{n}$ (some of which may be parallel to each other, and hence equal in $\mathcal{C}(F)$ ) and if these closed curves are marked by $\varepsilon_{1}, \ldots, \varepsilon_{n} \in\{+1,-1\}$, then let

$$
\Psi(S)=\sum_{i} \varepsilon_{i} \gamma_{i} \in \mathbb{Z} \mathcal{C}(F)
$$

Let $\mathcal{S}_{i j s}(D)$ be the set of enhanced states $S$ of $D$ with $i(S)=i, j(S)=j$, and $\Psi(S)=s$, and let $C_{i j s}(D)$ be the free abelian group spanned by enhanced states in $\mathcal{S}_{i j s}(D)$. Note that $C_{* * s}(D)=0$ if $s=\sum_{i=1}^{n} \varepsilon_{i} \gamma_{i}$ and the curves $\gamma_{i}, i=1, \ldots, n$, cannot be placed disjointly in $F$. On the other hand, if the curves $\gamma_{i}, i=1, \ldots, n$, can be placed disjointly in $F$, then $s$ represents a unique element of $\mathcal{B}(F)$. Therefore, alternatively, the index $s \in \mathbb{Z C}(F)$ can be replaced by $b \in \mathcal{B}(F)$.

\section{Differentials}

The definitions of the Khovanov chain groups of a link in $\mathbb{R}^{3}$ given in $\underline{\mathrm{K}} 1, \mathrm{BN}$, V] depend only on the choice of its diagram $D$. However, the definitions of differentials require an ordering of crossings of $D$. The same feature appears in 
our construction. Therefore, assume that the crossings of a link diagram $D$ in $F$ are ordered. Let the differential

$$
d_{i j s}: C_{i j s}(D) \longrightarrow C_{i-2, j, s}(D),
$$

be defined for enhanced states $S \in C_{i j s}(D)$ as follows

$$
d_{i j s}(S)=\sum_{v}(-1)^{t(S, v)} d_{v}(S)
$$

where the sum is over all crossings $v$ in $D$ and the "partial derivative" in the "direction" of $v$ is

$$
d_{v}(S)=\sum_{S^{\prime} \in \mathcal{S}(D)}\left[S: S^{\prime}\right]_{v} S^{\prime}
$$

The incidence number, $\left[S: S^{\prime}\right]_{v}$, is 1 if the following four conditions are satisfied:

(a) the crossing $v$ is marked by + in $S$ and by - in $S^{\prime}$,

(b) $S$ and $S^{\prime}$ assign the same markers to all the other crossings,

(c) the labels of the common circles in $S$ and $S^{\prime}$ are unchanged,

(d) $J(S)=J\left(S^{\prime}\right), \Psi(S)=\Psi\left(S^{\prime}\right)$.

Otherwise $\left[S: S^{\prime}\right]_{v}$ is equal to 0 .

The symbol $t(S, v)$ denotes the number of negative markers assigned to crossings in $S$ bigger than $v$.

For fixed $D, i, j, s$, we write

$$
\hat{d}_{v}(S)=(-1)^{t(S, v)} d_{v}(S)
$$

Hence

$$
d_{i j s}(S)=\sum_{\text {crossings } v \text { in } D} \hat{d}_{v}(S)
$$

for any $S \in \mathcal{S}_{i j s}(D)$.

If $\left[S: S^{\prime}\right]_{v}=1$ then $\tau(S)=\tau\left(S^{\prime}\right)+1$, and, therefore, $S$ and $S^{\prime}$ in a neighborhood of the crossing $v$ have one of the following forms, where $\varepsilon=+$ or - . 

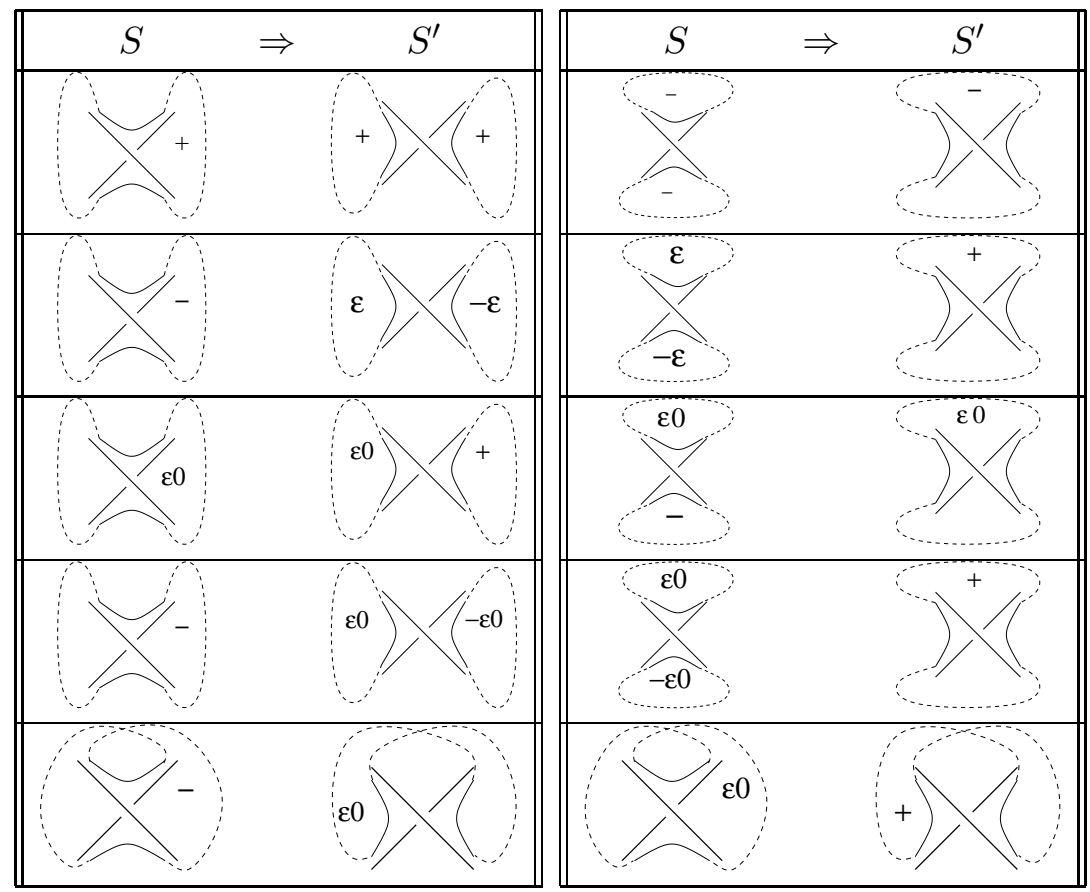

Table 2.1: Neighborhood of $v$

Our convention is to label the trivial circles by + or - and the non-trivial circles by +0 or -0 , to emphasize that the signs of the non-trivial circles do not count towards the $j$-grading. Notice that the states in last row labeled by $\varepsilon 0$ bound a Möbius band and, therefore, they may exist in unorientable surfaces only. The table has the following symmetry: it does not change by reversing all signs and markers together with flipping $S$ with $S^{\prime}$.

Note also that the degree of $d$ is -2 and that $C_{i, *, *}(D)=0$ either for all odd or all even $i$, depending on the number of crossings of $D$.

Finally, notice also that for any link diagram $D$ in $D^{2}, C_{i j s}(D)=0$ for $s \neq 0$ and that $C_{i, j, 0}(D)$ and $d$ coincide with Viro's $C_{i, j}(D)$ and $d,[V]$, for framed links.

\section{Khovanov homology}

\section{Theorem 5.1 (Proof in Section [11.2)}

(1) If $F \neq \mathbb{R P}^{2}$ and $D$ is an unoriented link diagram in $F$ with ordered crossings, then $d^{2}=0$. Hence $\left(C_{* j s}(D), d_{* j s}\right)$ is a chain complex for any $j \in \mathbb{Z}$, $s \in \mathbb{Z C}(F)$. 
(2) For any unoriented link diagram in $\mathbb{R P}^{2}$ with ordered crossings, $d^{2}=0 \bmod$ 2. Hence $\left(C_{* j s}(D) \otimes \mathbb{Z} / 2, d_{* j s}\right)$ is a chain complex for any $j \in \mathbb{Z}, s \in \mathbb{Z C}(F)$.

Theorem 5.1(1) does not hold for $\mathbb{R P}^{2}$.

Example 5.2 For the knot diagram $D$ in $\mathbb{R P}^{2}$ shown below, $C_{2,0,0}(D)$ is generated by the single enhanced state $S$ given by "+" markers at $v$ and $w$ and the single circle of $S$ labeled by "-",

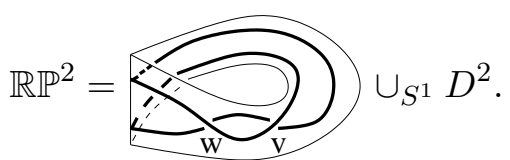

Since $d_{w} d_{v}(S)=0$ and $d_{v} d_{w}(S) \neq 0, d^{2}(S) \neq 0$.

From now on we assume that $F \neq \mathbb{R P}^{2}$. For any group $G$ we call the homology groups of $\left(C_{* j s}(D) \otimes G, d_{* j s}\right)$,

$$
H_{i j s}(D ; G)=\operatorname{Ker} d_{i j s} / \operatorname{Im} d_{i+2, j, s},
$$

the Khovanov homology groups of $D$ with coefficients in $G$.

For $G=\mathbb{Z}$ we simplify this notation to $H_{i j s}(D)$.

\section{Change of crossing order and Reidemeister moves}

Theorem 6.1 (Proof in Section [11.1) Given any two orderings of crossings of $D$ and the corresponding differentials $d_{1}, d_{2}$ on $C(D)$ there exists a natural chain isomorphism $f_{12}:\left(C(D), d_{1}\right) \rightarrow\left(C(D), d_{2}\right)$. The term "natural" means that for any three orderings of crossings of $D, f_{12} f_{23}=f_{13}$.

Theorem 6.2 (Proof in Sections 11.3, 11.4and11.5) Let $D$ be a link diagram in $F$.

(1) For any $i, j \in \mathbb{Z}, s \in \mathbb{Z C}(F), H_{i j s}(D)$ is preserved (up to an isomorphism) by the Reidemeister moves II and III.

(2) For a diagram $\supset$ obtained from $\supset$ by adding a negative twist, the $\operatorname{map} \stackrel{\varepsilon}{\mathcal{\varepsilon}} \rightarrow \varepsilon / \bigcirc$ for $\varepsilon= \pm, \pm 0$, induces a chain map $\rho_{I}: C_{i j s}(\curlyvee) \rightarrow$ $C_{i-1, j-3, s}(\bigcirc)$ which yields an isomorphism of homology groups,

$$
\rho_{I *}: H_{i j s}(\supset) \rightarrow H_{i-1, j-3, s}(\supset) .
$$

Consequently, if $M$ is an orientable $I$-bundle over $F$, then $H_{i j s}(D)$ is an invariant of (band) links in $M$ under ambient isotopy. 


\section{$7 \quad$ Viro's exact sequence}

For any skein triple

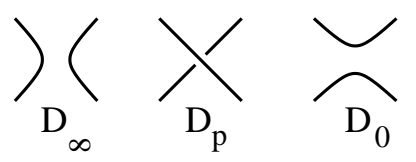

of link diagrams in a (possibly non-orientable) surface $F$ consider the map $\alpha_{0}: C_{i j s}\left(D_{\infty}\right) \rightarrow C_{i-1, j-1, s}\left(D_{p}\right)$ given by the embedding shown in Figure 7 1(a) and the map $\beta: C_{i j s}\left(D_{p}\right) \rightarrow C_{i-1, j-1, s}\left(D_{0}\right)$ which is the projection shown in Figure $71(\mathrm{~b})$.

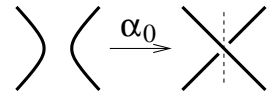

(a)

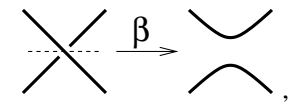

(b)

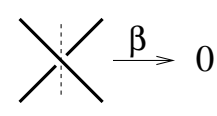

b)

Figure 1

The following is a generalization of the exact sequence of [V] Section 3.3].

Theorem 7.1 Let $F$ be any surface (including $\mathbb{R P}^{2}$ ) and let $D_{\infty}, D_{p}, D_{0}$ be a skein triple of any link diagrams in $F$ as above. Let the orderings of the crossings in $D_{\infty}$ and in $D_{0}$ be inherited from the ordering of crossings in $D_{p}$, and let $\alpha(S)=(-1)^{t^{\prime}(S)} \alpha_{0}(S)$ where $t^{\prime}(S)$ is the number of negatively labeled crossings in $S$ before $p$. Then

(1) the maps $\alpha: C_{i j s}\left(D_{\infty}\right) \rightarrow C_{i-1, j-1, s}\left(D_{p}\right), \beta: C_{i j s}\left(D_{p}\right) \rightarrow C_{i-1, j-1, s}\left(D_{0}\right)$ are chain maps, and

(2) the sequence

$$
0 \rightarrow C\left(D_{\infty}\right) \stackrel{\alpha}{\rightarrow} C\left(D_{p}\right) \stackrel{\beta}{\rightarrow} C\left(D_{0}\right) \rightarrow 0
$$

is exact.

Proof (1) Note that $\hat{d}_{p}(\alpha(S))=0$. (The partial derivative $\hat{d}_{p}$ was defined in Section 4.) Since $\left[S: S^{\prime}\right]_{q}=\left[\alpha_{0}(S): \alpha_{0}\left(S^{\prime}\right)\right]_{q}$ for any $q \neq p, \alpha$ and $\hat{d}_{q}$ commute up to sign. Furthermore,

$$
t^{\prime}(S)+t\left(\alpha_{0}(S), q\right)=t^{\prime}\left(S_{i}\right)+t(S, q) \bmod 2,
$$

for any summand $S_{i}$ of $\hat{d}_{q}(S)$ and, therefore, $\hat{d}_{q}(\alpha(S))=\alpha\left(\hat{d}_{q}(S)\right)$. Hence,

$$
d(\alpha(S))=\sum_{q \neq p} \hat{d}_{q}(\alpha(S))=\alpha\left(\sum_{q} \hat{d}_{q}(S)\right)=\alpha(d(S)) .
$$


We have $\beta\left(d_{p}(S)\right)=0$ and $d_{q}(\beta(S))=\beta\left(d_{q}(S)\right)$ for $S \in C\left(D_{p}\right)$ and any crossing $q$ of $D_{p}$. Since $t(\beta(S), q)=t(S, q)$ for any $q \neq p, \hat{d}_{q}(\beta(S))=\beta\left(\hat{d}_{q}(S)\right)$, and, hence, $\beta$ is a chain map as well.

(2) is obvious.

The short exact sequence (11) leads to the following long exact sequence of homology groups:

$$
\ldots \rightarrow H_{i j s}\left(D_{\infty}\right) \stackrel{\alpha_{*}}{\rightarrow} H_{i-1, j-1, s}\left(D_{p}\right) \stackrel{\beta_{*}}{\rightarrow} H_{i-2, j-2, s}\left(D_{0}\right) \stackrel{\partial}{\rightarrow} H_{i-2, j, s}\left(D_{\infty}\right) \rightarrow \ldots
$$

Let $\bar{\alpha}_{0}: C_{i j s}\left(D_{p}\right) \rightarrow C_{i+1, j+1, s}\left(D_{\infty}\right)$ and $\bar{\beta}: C_{i j s}\left(D_{0}\right) \rightarrow C_{i+1, j+1, s}\left(D_{p}\right)$ be the following homomorphisms of abelian groups:

$$
\bar{\beta}(\stackrel{\nearrow}{\smile})=\left(\frac{\nearrow}{\nearrow}\right) \text { and } \bar{\alpha}_{0}\left(\frac{\nearrow}{\nearrow}\right)=0, \bar{\alpha}_{0}\left(Y^{\prime}\right)=()() \text {. }
$$

Finally, let $\bar{\alpha}(S)=(-1)^{t^{\prime}(S)} \bar{\alpha}_{0}(S)$ and let $\gamma, \hat{\gamma}: C_{i j s}\left(D_{0}\right) \rightarrow C_{i, j+2, s}\left(D_{\infty}\right)$ be defined as

$$
\gamma=\bar{\alpha}_{0} d_{p} \bar{\beta} \quad \text { and } \quad \hat{\gamma}(S)=\bar{\alpha} \hat{d}_{p} \bar{\beta}=(-1)^{m(S)} \gamma(S),
$$

where $m(S)$ denotes the number of negative crossing markers in $S$.

The following properties of $\gamma$ and $\hat{\gamma}$ will be needed later:

Proposition 7.2 Let $F \neq \mathbb{R P}^{2}$.

(1) $\hat{\gamma}$ restricted to $\mathrm{Ker} d \subset C_{i j s}\left(D_{0}\right)$ defines the connecting homomorphism $\partial: H_{i j s}\left(D_{0}\right) \rightarrow H_{i, j+2, s}\left(D_{\infty}\right)$.

(2) $d \hat{\gamma}=-\hat{\gamma} d$.

(3) $\quad d \gamma=\gamma d$. Consequently, $\gamma: C_{i j s}\left(D_{0}\right) \rightarrow C_{i, j+2, s}\left(D_{\infty}\right)$ is a chain map. (Note, however, that neither $\bar{\alpha}_{0}$ nor $\bar{\beta}$ is a chain map.)

(4) For any enhanced state $S \in \mathcal{S}_{i j s}\left(D_{0}\right), \gamma(S)=\sum S^{\prime}$, where the sum is over all enhanced states $S^{\prime} \in \mathcal{S}_{i, j+2, s}\left(D_{\infty}\right)$ obtained from $S$ by changing the $\checkmark$-smoothing at $p$ to ) (-smoothing in such way that the circles which do not pass through the smoothings of $p$ in $S$ and $S^{\prime}$ have identical labels.

Proof (1) Since $\bar{\alpha} \hat{d}_{q} \bar{\beta}=0$ for $q \neq p, \hat{\gamma}=\bar{\alpha} \hat{d}_{p} \bar{\beta}=\bar{\alpha} d \bar{\beta}$.

Since $\bar{\alpha}$ and $\bar{\beta}$ split the exact sequence of groups (11), $\hat{\gamma}$ restricted to Kerd defines the connecting differential $\partial$.

(2) Notice that

$$
\hat{d}_{q}(\bar{\beta}(S))=\bar{\beta}\left(\hat{d}_{q}(S)\right) \quad \text { and } \quad \hat{d}_{q}(\bar{\alpha}(S))=\bar{\alpha}\left(\hat{d}_{q}(S)\right) \quad \text { for } q \neq p .
$$


The second equality follows from

$$
t^{\prime}\left(S_{q}\right)+t(S, q)=t^{\prime}(S)+t\left(\bar{\alpha}_{0}(S), q\right) \bmod 2,
$$

where $S_{q}$ is any state such that $\left[S: S_{q}\right]_{q}=1$. By equation (12) in Section 11.2 $d_{p}$ and $d_{q}$ commute and, therefore, $\hat{d}_{p} \hat{d}_{q}=-\hat{d}_{q} \hat{d}_{p}$. Since $\bar{\alpha} \hat{d}_{q} \bar{\beta}=0$ for $q \neq p$,

$$
d \hat{\gamma}(S)=d\left(\bar{\alpha} \hat{d}_{p} \bar{\beta}(S)\right)=\sum_{q} \hat{d}_{q} \bar{\alpha} \hat{d}_{p} \bar{\beta}(S)=-\sum_{q} \bar{\alpha} \hat{d}_{p} \bar{\beta}\left(\hat{d}_{q} S\right)=-\hat{\gamma} d(S) .
$$

(3) follows from (2) by assuming that $p$ is the highest (i.e. the last) of the vertices of $D_{p}$.

(4) is left to the reader.

Note that the definitions of $\gamma, \hat{\gamma}$ do not depend on the position of $p$ in the ordering of vertices of $D_{p}$. In fact, by Proposition 7.2(4), these maps can be defined without referring to $D_{p}$ at all.

\section{Categorification of the skein modules of $I$-bundles over surfaces}

Khovanov homology groups, $H_{i j}(L)$, for $L \subset \mathbb{R}^{3}$, normalized according to $\mathrm{V}$ ] (for unoriented, framed links), satisfy the following equation

$$
\chi_{A}\left(H_{* *}(L)\right)=[L],
$$

where [.] is the Kauffman bracket normalized by $[\emptyset]=1$, and $\chi_{A}\left(H_{* *}\right)$ is a version of the polynomial Euler characteristic defined for the purpose of this paper as

$$
\chi_{A}\left(H_{* *}\right)=\sum_{i, j} A^{j}(-1)^{\frac{j-i}{2}} r k H_{i j} .
$$

Before formulating a version of identity (5) for links in an $I$-bundle $M$ over a surface $F$, one must realize that the natural generalization of the Kauffman bracket for band links $L \subset M$ is their representation in the skein module of $M,[L] \in \mathcal{S}_{b}\left(M ; \mathbb{Z}\left[A^{ \pm 1}\right]\right)$. The bracket $[L]$ can be identified with a polynomial only if $\mathcal{S}_{b}\left(M ; \mathbb{Z}\left[A^{ \pm 1}\right]\right)=\mathbb{Z}\left[A^{ \pm 1}\right]$, for example for $L \subset D^{2} \times I$ and $L \subset S^{2} \times I$. Nonetheless, by Theorem 2.1] $[L]$ presents uniquely as

$$
[L]=\sum_{b \in \mathcal{B}(F)} p_{b}(L) b
$$


and, therefore, the "Kauffman bracket" of $L$ can be thought as the set of polynomials $p_{b}(L) \in \mathbb{Z}\left[A^{ \pm 1}\right]$ determined by the above equation. We are going to show that polynomial Euler characteristics of our homology groups $H_{i j s}(L)$ determine $p_{b}(L)$ for all $b \in \mathcal{B}(F)$, if $F$ is orientable. In other words, our homology groups "categorify" the coefficients of links expressed in the natural bases of the skein modules of $F \times I$ for orientable surfaces $F$. A slightly weaker statement holds also for twisted $I$-bundles over unorientable surfaces.

Note that the group $G=\prod_{\mathcal{C}(F)}\{ \pm 1\}$ acts on $\mathbb{Z C}(F)$, by

$$
\left(\varepsilon_{\gamma}\right) \cdot \sum_{\gamma \in \mathcal{C}(F)} c_{\gamma} \gamma \rightarrow \sum_{\gamma \in \mathcal{C}(F)} \varepsilon_{\gamma} c_{\gamma} \gamma
$$

Furthermore, it is not difficult to prove that

$$
H_{i, j, g s}(D)=H_{i, j, s}(D) \text {, for any } g \in G, s \in \mathbb{Z C}(F) \text {, and } i, j \in \mathbb{Z} \text {. }
$$

Hence it is enough to consider $s=\sum c_{\gamma} \gamma$, with $c_{\gamma} \geq 0$. Such elements form the semigroup $\mathbb{N C}(F)$, where $\mathbb{N}=\{0,1, \ldots\}$. Since $\mathcal{B}_{n b}(F)$ embeds into $\mathbb{N C}(F)$ in a natural way, one might expect that $p_{b}(L)$, for $b \in \mathcal{B}_{n b}(F)$, is the polynomial Euler characteristic of $H_{* * b}(L)$, but that is not the case. In order to explicate the relationship between these polynomials we need to consider the ring of Laurent polynomials whose set of formal variables $x_{\gamma}$ is in $1-1$ correspondence with elements of $\mathcal{C}(F)$. Consider a map

$$
\phi:\{\text { non-trivial simple closed curves in } F\} \rightarrow \mathbb{Z}\left[x_{\gamma}^{ \pm 1}: \gamma \in \mathcal{C}(F)\right],
$$

sending each $\gamma \in \mathcal{C}(F)$ to $x_{\gamma}+x_{\gamma}^{-1}$, and sending every curve $\gamma$ bounding a Möbius band to $\phi(\gamma)=2$. This map extends multiplicatively to

$$
\phi: \mathcal{B}(F) \rightarrow \mathbb{Z}\left[x_{\gamma}^{ \pm 1}: \gamma \in \mathcal{C}(F)\right]
$$

if $b=\bigcup_{i=1}^{d} \gamma_{i}$, where $\gamma_{i}$ are (possibly parallel) non-trivial simple closed curves in $F$, then $\phi(b)=\prod_{i=1}^{d} \phi\left(\gamma_{i}\right)$. Finally, by Theorem 2.1 $\phi$ extends to

$$
\phi: \mathcal{S}\left(M ; \mathbb{Z}\left[A^{ \pm 1}\right]\right)=\mathbb{Z}\left[A^{ \pm 1}\right] \mathcal{B}(F) \rightarrow \mathbb{Z}\left[A^{ \pm 1}, x_{\gamma}^{ \pm 1}: \gamma \in \mathcal{C}(F)\right]
$$

in an obvious way.

Hence we have $\phi([L])=\sum q_{m}(L) m$, where the sum is over all monomial $2 \frac{2}{2} m$ in variables $x_{\gamma}^{ \pm 1}$, for $\gamma \in \mathcal{C}(F)$, and $q_{m}(L) \in \mathbb{Z}\left[A^{ \pm 1}\right]$. Note that the set of such monomials can be identified with $\mathbb{Z C}(F)$.

Theorem 8.1 For any $L$ in an orientable $I$-bundle over $F$ and for any $s \in$ $\mathbb{Z C}(F), \chi_{A}\left(H_{* * s}(L)\right)=q_{s}(L)$.

\footnotetext{
${ }^{2}$ By a monomial we mean here a product of variables with leading coefficient 1 .
} 
Proof By the Kauffman bracket skein relations,

$$
\begin{gathered}
\phi([\searrow])=A \phi([\smile])+A^{-1} \phi([)(]), \\
\phi([L \cup \bigcirc])=-\left(A^{2}+A^{-2}\right) \phi([L]),
\end{gathered}
$$

and, consequently,

$$
q_{s}(\lambda)=A q_{s}(\bumpeq)+A^{-1} q_{s}(\supset), \quad q_{s}(L \cup \bigcirc)=-\left(A^{2}+A^{-2}\right) q_{s}(L) .
$$

Additionally, if $L$ is a link diagram in $F$ and $M \subset F$ is curve disjoint from $L$ bounding a Möbius band then $\phi([L \cup M])=2 \phi([L])$, and consequently, $q_{s}(L \cup M)=2 q_{s}(L)$.

On the other hand, (6) and (2) imply

$$
\chi_{A}\left(H_{* * s}(\searrow)\right)=A \chi_{A}\left(H_{* * s}(\asymp)\right)+A^{-1} \chi_{A}\left(H_{* * s}(\supset)\right) .
$$

Additionally, since

$$
H_{i j s}(L \cup \bigcirc)=H_{i, j+2, s}(L) \oplus H_{i, j-2, s}(L),
$$

we have

$$
\chi_{A}\left(H_{* * s}(L \cup \bigcirc)\right)=-\left(A^{2}+A^{-2}\right) \chi_{A}\left(H_{* * s}(L)\right) .
$$

Finally, if $L$ is a link diagram in $F$ and $M \subset F$ is curve disjoint from $L$ bounding a Möbius band then

$$
H_{i j s}(L \cup M)=H_{i j s}(L) \oplus H_{i j s}(L)
$$

and

$$
\chi_{A}\left(H_{* * s}(L \cup M)\right)=2 \chi_{A}\left(H_{* * s}(L)\right) .
$$

By above equations, it is enough to prove the statement for links in $\mathcal{B}_{n b}(F)$. Assume hence that $L$ has a diagram composed of $n_{i}>0$ curves $\gamma_{i}$ (not parallel to each other) for $i=1, \ldots, d$. Since the diagram of $L$ has no crossings, we have $H_{i j s}(L)=C_{i j s}(L)$ and $H_{i j s}(L)=0$ for $(i, j) \neq(0,0)$. Consequently,

$$
\chi_{A}\left(H_{*, *, s}(L)\right)=\operatorname{rank} C_{0,0, s}(L) .
$$

If $s=\sum_{i=1}^{d} k_{i} \gamma_{i}$ then (8) is the number of labelings of components of $L$ by signs \pm such that the sum of signs of $n_{i}$ parallel components $\gamma_{i}$ is $k_{i}$. This is precisely the number of monomials $x_{\gamma_{1}}^{k_{1}} \ldots x_{\gamma_{d}}^{k_{d}}$ appearing in the total expansion of

$$
\prod_{1}^{d}\left(x_{\gamma_{i}}+x_{\gamma_{i}}^{-1}\right)^{n_{i}} .
$$

Since the groups $H_{i j s}(L)$ determine the polynomials $q_{s}(L)$ and $\phi$ is an embedding for an orientable $F$, we get 
Corollary 8.2 If $F$ is orientable then the homology groups $H_{i j s}(L)$, taken over all $s \in \mathbb{Z C}(F), i, j \in \mathbb{Z}$, determine the polynomials $p_{b}(L)$ for $b \in \mathcal{B}(F)$.

More specifically, for any $s=\sum_{i=1}^{d} k_{i} \gamma_{i}$ let $\lambda(s)=\prod_{i=1}^{d}\left(\frac{y_{i}+\sqrt{y_{i}^{2}-4}}{2}\right)^{k_{i}}$.

Then $p_{s}(L)$ is the coefficient of the monomial $y_{1}^{k_{1}} \ldots y_{d}^{k_{d}}$ in the expansion of $\sum_{s} q_{s}(L) \lambda(s)$.

If $F$ is unorientable, then $\phi$ is not an embedding and the statement of Corollary 8.2 does not hold. In this case, for any $b \in \mathcal{B}_{n b}(F)$, let $\mathcal{B}(F ; b)$ denote the set of all basis elements of $F$ obtained by adding to $b$ disjoint closed curves bounding Möbius bands. We have

$$
\mathcal{B}(F)=\amalg_{b \in \mathcal{B}_{n b}(F)} \mathcal{B}(F ; b)
$$

and the following generalization of Corollary 8.2 holds:

Corollary 8.3 For any orientable $I$-bundle over $F$ the homology groups $H_{i j s}(L)$, taken over all $s \in \mathbb{Z C}(F), i, j \in \mathbb{Z}$, determine the sums

$$
\sum_{b^{\prime} \in \mathcal{B}(F ; b)} 2^{\left|b^{\prime}\right|-|b|} p_{b^{\prime}}(L)
$$

for any $b \in \mathcal{B}_{n b}(F)$. Here, $|b|$ denotes the number of components of $b$.

\section{Cohomology}

For a link diagram $D$ in $F$, the Khovanov cohomology groups $H_{i j s}(D)$ are the cohomology groups of the cochain complex $\left(C^{* * *}(D), d^{*}\right)$, for $C^{* * *}(D)=$ $\operatorname{Hom}\left(C_{* * *}(D), \mathbb{Z}\right)$. Here is another way of defining the cochain complex of $D$ :

Proposition 9.1 The following diagram commutes:

$$
\begin{aligned}
& C_{i j s}(D) \stackrel{\tilde{d}}{\longrightarrow} C_{i+2, j, s}(D) \\
& \downarrow \psi \quad \downarrow \psi \\
& C^{i j s}(D) \stackrel{d^{*}}{\longrightarrow} C^{i+2, j, s}(D),
\end{aligned}
$$

where $\tilde{d}$ is defined by

$$
\tilde{d}(S)=\sum_{\substack{\text { all states } S^{\prime} \\ \text { all crossings } v \text { in } D}}(-1)^{t(S, v)}\left[S^{\prime}: S\right]_{v} S^{\prime},
$$

and $\psi$ is the isomorphism given by the identification of the basis composed of enhanced states with its dual. 
Proof Denote the dual of $x \in C(D)$ by $x^{*} \in C^{*}(D)$. We need to prove that $(\tilde{d}(S))^{*}=d^{*}\left(S^{*}\right)$, for any $S \in C(D)$. Equivalently, that for any $S_{0} \in C(D)$,

$$
(\tilde{d}(S))^{*}\left(S_{0}\right)=d^{*}\left(S^{*}\right)\left(S_{0}\right) .
$$

The left side of this equation expands to

$$
\sum_{\substack{\text { all states } \\ \text { all crossings } v \text { in } D}}(-1)^{t(S, v)}\left[S^{\prime}: S\right]_{v}\left(S^{\prime}\right)^{*}\left(S_{0}\right)=(-1)^{t\left(S, v_{0}\right)}\left[S_{0}: S\right]_{v_{0}},
$$

where $v_{0}$ is the crossing of $D$ at which the markers of $S_{0}$ and $S$ differ. If $\left[S_{0}: S\right]_{v}=1$ for some $v$ then such crossing $v$ is unique and $v_{0}=v$. On the other hand, we have

$$
d^{*}\left(S^{*}\right)\left(S_{0}\right)=S^{*}\left(d\left(S_{0}\right)\right)=(-1)^{t\left(S_{0}, v_{0}\right)}\left[S_{0}: S\right]_{v_{0}} .
$$

Since the markers of $S$ and $S_{0}$ differ at $v_{0}$ only, $t\left(S_{0}, v_{0}\right)=t\left(S, v_{0}\right)$ and the proof is complete.

For a link diagram $D$ we denote its mirror image, i.e. the diagram obtained by switching all crossings of $D$, by $\bar{D}$. In order to relate the cohomology of $D$ to homology of $\bar{D}$, consider $d^{+}(S)=\sum_{v}(-1)^{t^{+}(S, v)} d_{i j s ; v}(S)$, where $t^{+}(S, v)$ is the number of positive markers assigned to crossings in $S$ bigger than $v$. We can show that $d^{+} d^{+}(S)=0$ in the same way as $d^{2}(S)=0$ - both statements follow from equation (12) in Section 11.2, Therefore either of the differentials $d, d^{+}$could be used for the definition of the Khovanov chain complex of links. The following lemma shows that these chain complexes are isomorphic.

Proposition 9.2 Let $D$ be a link diagram with $n$ crossings and let for any enhanced state $S$ of $D, g(S)=(-1)^{u(S)} S$, where $u(S)$ is the number of positively marked crossings $v_{i}$ in $S$ such that $i=n+1 \bmod 2$. This map induces an isomorphism of chain complexes $g:(C(D), d) \rightarrow\left(C(D), d^{+}\right)$.

Proof Both $d(S)$ and $d^{+}(S)$ are linear combinations of states $S^{\prime}$ obtained from $S$ by changing one positive marker to a negative one. Therefore it is enough to prove that if $S, S^{\prime}$ differ by a marker at an $i$ th vertex $v_{i}$ then the following morphisms form a commutative diagram:

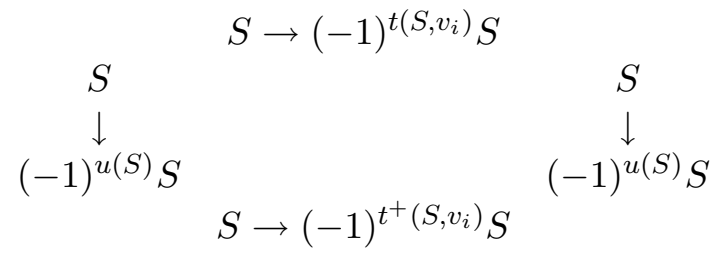

Since $t^{+}\left(S, v_{i}\right)=n-i-t\left(S, v_{i}\right)$ and $u(S)-u\left(S^{\prime}\right)=n-i \bmod 2$ the above diagram commutes. 
For a crossing $v$ in $D$, we denote by $\bar{v}$ the corresponding crossing in $\bar{D}$. We order the crossings of $D$ and of $\bar{D}$ in a consistent manner, i.e. if $v$ is the $i$ th crossing in $D$ then $\bar{v}$ is the $i$ th crossing in $\bar{D}$. Now, consider the map

$$
\varphi: C_{i j s}(D) \rightarrow C_{-i,-j,-s}(\bar{D})
$$

such that for any enhanced state $S$ of $D, \varphi(S)$ is an enhanced state of $\bar{D}$ whose every crossing $\bar{v}$ is labeled by the marker opposite to the marker of $v$ in $S$. Under these assumptions the loops of $S$ and $\varphi(S)$ are naturally identified. We assume that $\varphi(S)$ reverses the signs of all loops in $S$.

Proposition 9.3 The following diagram commutes

$$
\begin{array}{ccc}
C_{i j s}(D) & \stackrel{\tilde{d}}{\downarrow} & C_{i+2, j, s}(D) \\
\downarrow \varphi & & \downarrow \varphi \\
C_{-i,-j,-s}(\bar{D}) & \stackrel{d^{+}}{\longrightarrow} & C_{-i-2,-j,-s}(\bar{D}) .
\end{array}
$$

Proof We need to prove that

$$
\varphi(\tilde{d}(S))=d^{+}(\varphi(S)), \text { for any } S \in C(D) .
$$

The left hand side equals

$$
\sum_{\substack{\text { all } S^{\prime} \in \mathcal{S}(D) \\ \text { all crossings } v \text { in } D}}(-1)^{t(S, v)}\left[S^{\prime}: S\right]_{v} \varphi\left(S^{\prime}\right)
$$

and the right hand of (9) is

$$
\sum_{\substack{\text { all } \bar{S} \in \mathcal{S}(\bar{D}) \\ \text { all crossings } \bar{v} \text { in } \bar{D}}}(-1)^{t^{+}(\varphi(S), \bar{v})}[\varphi(S): \bar{S}]_{\bar{v}} \bar{S} .
$$

Since the states $\bar{S}$ of $\bar{D}$ are in 1-1 correspondence with states of $D$ via the map $\varphi$, this expression takes the form

$$
\sum_{\substack{\text { all } S^{\prime} \in \mathcal{S}(D) \\ \text { all crossings } v \text { in } D}}(-1)^{t^{+}(\varphi(S), \bar{v})}\left[\varphi(S): \varphi\left(S^{\prime}\right)\right]_{\bar{v}} \varphi\left(S^{\prime}\right) .
$$

Now the statement follows from the following two identities:

$$
\begin{gathered}
t(S, v)=t^{+}(\varphi(S), \bar{v}) \\
{[S: T]_{v}=[\varphi(T): \varphi(S)]_{\bar{v}} \text { for any } S, T \in \mathcal{S}(D) .}
\end{gathered}
$$

The first one follows from the fact that $\varphi$ reverses the signs of markers, and the second one follows directly from the definition of the incidence number. 
By combining the statements of Propositions 9.1, 9.2, and 9.3, we conclude

Corollary 9.4 For any diagram $D$ and $i, j \in \mathbb{Z}, s \in \mathbb{Z C}(F)$,

$$
g_{*} \varphi_{*} \psi_{*}^{-1}: H^{i j s}(D) \rightarrow H_{-i,-j,-s}(\bar{D})
$$

is an isomorphism.

By Universal Coefficient Theorem (cf. [H, Corollary 3.5]),

$$
H_{-i,-j,-s}(\bar{D})=H^{i j s}(D)=H_{i j s}(D) / T_{i j s}(D) \oplus T_{i-2, j, s}(D),
$$

where $T_{i j s}(D)$ is the torsion part of $H_{i j s}(D)$. Consequently, the torsions of homology groups of $D$ and $\bar{D}$ coincide (up to a shift of indices). This observation is used in $\mathrm{AP}$.

\section{Khovanov homology for different $I$-bundle struc- tures}

Any handlebody $H$ is an $I$-bundle over a surface, although the $I$-bundle structure of $H$ is not unique. For example, if

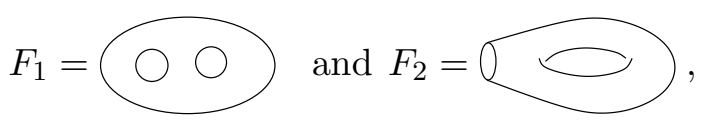

then $F_{1} \times I \simeq F_{2} \times I$ are both handlebodies of genus 2 . Hence, it is natural to ask whether there exist homology groups of links $L \subset H$ which do not depend on the $I$-bundle structure of $H$ and on the choice of $s \in \mathbb{Z C}(F)$ for the base surface $F$. One obvious choice to consider is

$$
\mathcal{H}_{i j}(L)=\bigoplus_{s \in \mathbb{Z C}(F)} H_{i j s}(L)
$$

We are going to give two examples showing that unfortunately $\mathcal{H}_{i j}(L)$ does depend on the choice of $I$-bundle structure of $H$.

Example 1 The spaces $F_{2} \times I$ and $F_{1} \times I$ are homeomorphic by a homeomorphism mapping the curve $\gamma$ in $F_{2} \times\{0\}$ to the curve $\gamma^{\prime}$ in $\partial\left(F_{1} \times I\right)$.

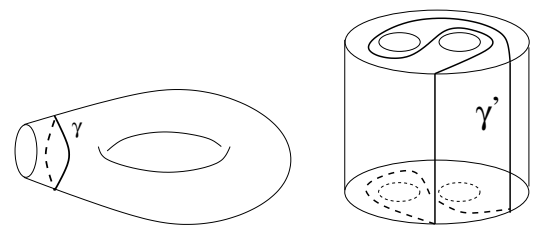


Therefore, the knot $K$ represented by the diagram $D=\gamma$ in $F_{2}$ is given by the following diagram $D^{\prime}$ in $F_{1}$,

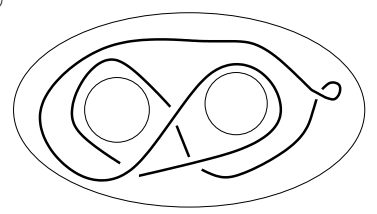

The calculations of homology groups of $D$ and $D^{\prime}$ yield

$$
\begin{gathered}
\mathcal{H}_{i j}(D)= \begin{cases}\mathbb{Z}^{2} & \text { for }(i, j)=(0,0) \\
0 & \text { otherwise }\end{cases} \\
\mathcal{H}_{i j}\left(D^{\prime}\right)= \begin{cases}\mathbb{Z}^{7} & \text { for }(i, j)=(0,-2) \\
\mathbb{Z}^{8} & \text { for }(i, j)=(-2,-4) \\
\mathbb{Z}^{3} & \text { for }(i, j)=(-4,-6) \\
0 & \text { otherwise. }\end{cases}
\end{gathered}
$$

Example 2 The homeomorphism of $H_{2}=F_{1} \times I$ given by the following Dehn twist

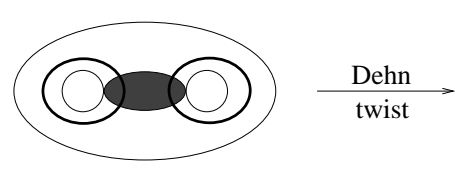

$\mathrm{D}$

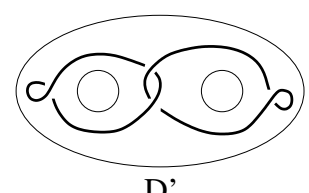

D'

maps $D$ to $D^{\prime}$.

We have

$$
\begin{gathered}
\mathcal{H}_{i j}(D)=\left\{\begin{array}{ll}
\mathbb{Z}^{4} & \text { for }(i, j)=(0,0) \\
0 & \text { otherwise }
\end{array}\right. \text { and } \\
\mathcal{H}_{i j}\left(D^{\prime}\right)= \begin{cases}\mathbb{Z}^{4} & \text { for }(i, j)=(3,5) \\
\mathbb{Z}^{2} & \text { for }(i, j)=(1,3) \text { and }(-1,-1) \\
0 & \text { otherwise. }\end{cases}
\end{gathered}
$$

\section{The proofs}

\subsection{Invariance of homology under crossing order changes}

Consider two different orderings of the vertices of a link diagram $D$. Let $S$ be an enhanced state of $D$. Each of the orderings induces an ordering on the set of 
negatively marked crossings in $S$. These two orderings (restricted to negative crossings) differ by a permutation $\sigma_{S}$. We define $f_{12}(S)=\operatorname{sgn}\left(\sigma_{S}\right) S$ and extend it to $f_{12}: C(D) \rightarrow C(D)$.

For any three orderings of crossings of $D, f_{12} f_{23}=f_{13}$ and therefore for the remainder of the proof we assume that the orderings of crossings of $D$ differ by a transposition $(i, i+1)$. We need to prove that $f_{12}\left(d_{1}(S)\right)=d_{2}\left(f_{12}(S)\right)$, and for that it is enough to show that if $\left[S: S^{\prime}\right]_{v}=1$ then

$$
(-1)^{t_{1}(v, S)-t_{2}(v, S)}=\operatorname{sgn}(\sigma) \operatorname{sgn}\left(\sigma^{\prime}\right),
$$

where $t_{1}(v, S), t_{2}(v, S)$ denote the numbers of negative markers assigned to vertices above $v$ in $S$, with respect to the two orderings. Since $\left[S: S^{\prime}\right]_{v}=1$, $v$ has the + marker in $S$. To complete the proof note that each of the sides of this equation is equal -1 if and only if either

- $v$ is the $i$ th crossing in $D$ (with respect to the first ordering) and $S$ assigns - to the $(i+1)$ st crossing; or

- $v$ is the $(i+1)$ th crossing in $D$ and $S$ assigns - to the $i$ th crossing.

\subsection{Proof of $d^{2}=0$ (Theorem 5.1)}

For any link diagram $D$ in $F$, any $i, j \in \mathbb{Z}, s \in \mathbb{Z C}(F)$, and any enhanced state $S \in C_{i, j, s}(D)$, we are going to prove that $d_{* j s}^{2}(S)=0$, if $F \neq \mathbb{R P}^{2}$, and $d_{* j s}^{2}(S)=0 \bmod 2$ for any $F$. The signs of partial derivatives in $d_{i j s}$ are defined in such a way that it is sufficient to show the following two equations

$$
\begin{gathered}
d_{v} \circ d_{w}(S)=d_{w} \circ d_{v}(S) \text { if } F \neq \mathbb{R P}^{2} \\
d_{v} \circ d_{w}(S)=d_{w} \circ d_{v}(S) \bmod 2(\text { for any } F)
\end{gathered}
$$

for any crossings $v$ and $w$ of $D$.

Let $D^{\prime}$ be a diagram obtained from $D$ by smoothing all the crossings of $D$ according to $S$, except for $v$ and $w$. Since $S$ can be considered as a state of $D^{\prime}$, it is sufficient to show equation (12) for $D^{\prime}$. Therefore, from now on we assume that $D$ has 2 crossings only: $v$ and $w$.

Lemma 11.1 If (12), (13) hold for all states of $D$ then they hold for all states of $\bar{D}$ as well.

Proof If $d^{2}(S)=0$ for all $S \in C_{2, *, *}(D)$, then $\left(d^{*}\right)^{2}\left(S^{*}\right)=0$ for all $S^{*} \in$ $C^{-2, *, *}(D)$. Hence, by Theorems 9.1 and 9.3, $d^{2}(S)=0$ for all $S \in C_{2, *, *}(\bar{D})$. 
We denote the trivial circles in any state by $\mathrm{T}$ and the nontrivial circles by $\mathrm{N}$. Hence NT denotes two circles, one trivial and one nontrivial. Our proof of (12) and (13) does not use the fact that $D$ lies in a surface and it relies entirely on the following principle: If $F \neq \mathbb{R P}^{2}$ then the change of smoothing of any crossing of $D$ results in one of the following transformations:

$$
\begin{gathered}
\mathrm{T} \rightarrow \mathrm{N}, \mathrm{TT} \text { or NN, } \mathrm{N} \rightarrow \mathrm{T}, \mathrm{N}, \mathrm{NT} \text { or } \mathrm{NN} \\
\mathrm{TT} \rightarrow \mathrm{T}, \quad \mathrm{NN} \rightarrow \mathrm{T} \text { or } \mathrm{N}, \quad \mathrm{TN} \rightarrow \mathrm{N} .
\end{gathered}
$$

Additionally, if $F=\mathbb{R P}^{2}$ then another possible transformation is

$$
\mathrm{T} \rightarrow \mathrm{T} .
$$

(This transformation may not happen in $F \neq \mathbb{R P}^{2}$. Proof: A trivial circle bounds a disk, which after the change of smoothing becomes a Möbius band. If the boundary of Möbius band in $F$ is trivial then $F$ is a union of a Möbius band and a disk.)

Since our proof does not use any information about the position of $D$ in $F$, we can think of any 2 crossing link diagram in $F$ as an abstract 4-valent graph with 2 rigid vertices. Any such graph is obtained by connecting the 1-valent vertices in the picture below:

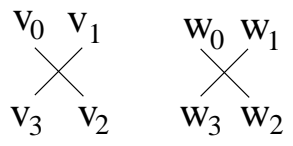

Hence we consider all possible pairings of the following eight points $v_{0}, v_{1}, v_{2}$, $v_{3}, w_{0}, w_{1}, w_{2}, w_{3}$. Since obviously (12), (13) hold for diagrams $D$ in which $v$ and $w$ belong to different connected components, without loss of generality we assume that $v_{0}, w_{0}$ are connected by an arc. The following operations identify isomorphic graphs:

(1) exchanging $v$ with $w$;

(2) cyclic permutation $v_{i} \rightarrow v_{i-k} \bmod 4$ and $w_{i} \rightarrow w_{i-k \bmod 4}$ for all $i$; Since $v_{0}$ and $w_{0}$ are always assumed to be connected, this operation is allowed only if $v_{k}$ and $w_{k}$ are connected.

(3) flip: $v_{i}$ is exchanged with $v_{i+2}$ for some $i \in\{0,1,2,3\}$ while $w_{i+1} \bmod 4$ and $w_{i+3} \bmod 4$ are fixed. An analogous flip is allowed for vertex $w$ as well. 
The last relation follows from the fact that if link diagrams $D_{1}, D_{2}$ are represented by graphs $\Gamma_{1}, \Gamma_{2}$ related by a flip, then there is a natural $1-1$ correspondence between the states of $D_{1}$ and the states of $D_{2}$. This correspondence is preserved by changing of smoothing, incidence numbers, and partial derivatives.

The proof of the following lemma is left to the reader:

Lemma 11.2 Up to the above relations, there are precisely 5 different abstract connected 4 -valent graphs with 2 rigid vertices:

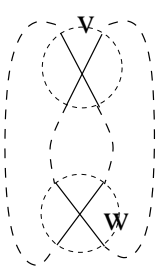

1

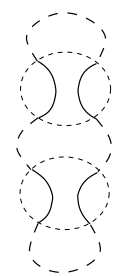

2

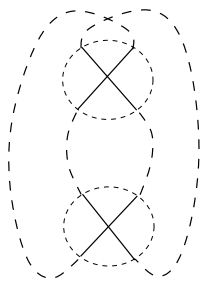

3

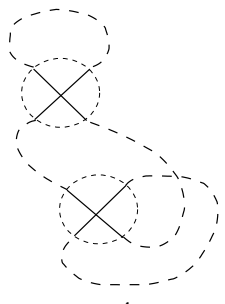

4

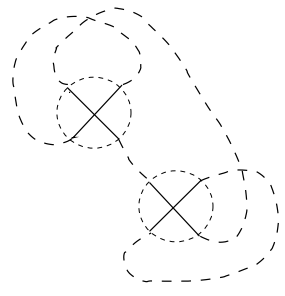

5

We prove (12) and (13) for the above graphs on a case by case basis. In the proofs we will frequently refer to the following arguments for $S$ :

(NZ) We assume that either $d_{v} d_{w}(S)$ or $d_{w} d_{v}(S)$ is non-zero. (Otherwise, clearly (12), (13) holds.)

$(\tau)$ If $d_{v}(S)=\sum_{i} S_{i}, d_{w}\left(S_{i}\right)=\sum_{j} S_{i j}$ then $\tau\left(S_{i}\right)=\tau(S)+1, \tau\left(S_{i j}\right)=\tau(S)+2$ for all $i, j$. (Analogous statement for $v$ and $w$ exchanged).

(S) Argument by the symmetry of the picture.

Notation In the proof we use $\underbrace{T \ldots T}_{k} \underbrace{N \ldots N}_{l}$ to denote any state composed of $k$ trivial and $l$ nontrivial circles. When labels of circles are of importance we use the symbols $\odot,-(-), \varepsilon$ to denote trivial components labeled by,+- , and $\varepsilon$, respectively; $\varepsilon$ denotes + or - . The symbols $\oplus 0,-0$ are used to denote the nontrivial circles, labeled by + and - , respectively. By abuse of notation, we sometimes write $\mathrm{S}=\mathrm{TN}$ or $\mathrm{S}=-(+0$ despite the fact that neither $\mathrm{TN}$ and $\odot \oplus 0$ denotes a unique state. In particular, they do not specify which of the circles of $\mathrm{S}$ is trivial. If the order of circles of $S$ is important and if the circles of $S$ are positioned one above another, then we use ${ }_{(0)}$ to specify that the circle above is trivial and labeled by - and the bottom one is non-trivial and labeled by + . 


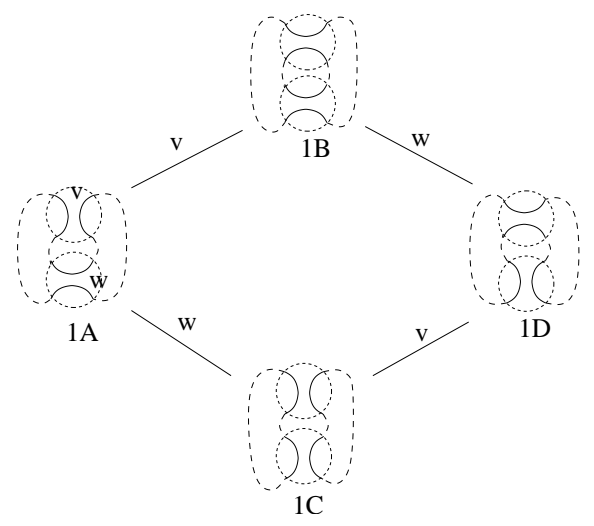

We are going to prove (1213) for states of the above four types, case by case. In each of these cases, the edges with appropriate orientation will represent the partial derivatives. For example, if $S$ if of type 1A then the edges are oriented outwards 1A. Notice that $d_{v}(S)$ is a sum of states of type 1B, etc.

(1A) By $(\mathrm{NZ}, \tau), 1 \mathrm{~A}=\odot, 1 \mathrm{D}=\oplus$. Hence $1 \mathrm{~A}$ is a trivial loop, and therefore each of the states $1 \mathrm{~B}$ and $1 \mathrm{C}$ is either composed of two trivial circles or two nontrivial circles. In either case $d_{v} d_{w}(\odot)=d_{w} d_{v}(\odot)=2 \odot$.

(1B) If $1 \mathrm{~A}$ and $1 \mathrm{~B}$ are either both trivial or both nontrivial then by (S), (12) holds. Hence, again by $(\mathrm{S})$, we assume that $1 \mathrm{~A}$ is $\mathrm{T}$ and $1 \mathrm{D}$ is $\mathrm{N}$, and therefore $1 \mathrm{~B}$ and $1 \mathrm{C}$ are NN. Now by $(\tau)$, both sides of (12) are 0 .

(1C) and (1D) The proof follows from (1A) and (1B) by Lemma 11.1. (Alternatively, the proof follows here from symmetry of the above diagram.)

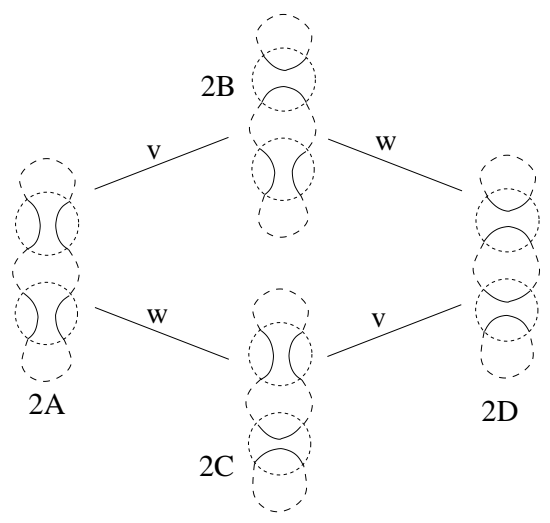

(2A) If $2 \mathrm{D}$ is $\mathrm{TTT}$ then $2 \mathrm{~B}, 2 \mathrm{C}$ are $\mathrm{TT}$ and the statement follows by (S). Hence assume that $2 \mathrm{D}$ is not TTT. Furthermore, by $(\tau)$ and (NZ) we assume that if $2 \mathrm{~A}$ is $\mathrm{N}$ then $2 \mathrm{D}$ is TTN. Therefore, we have the following possibilities: 


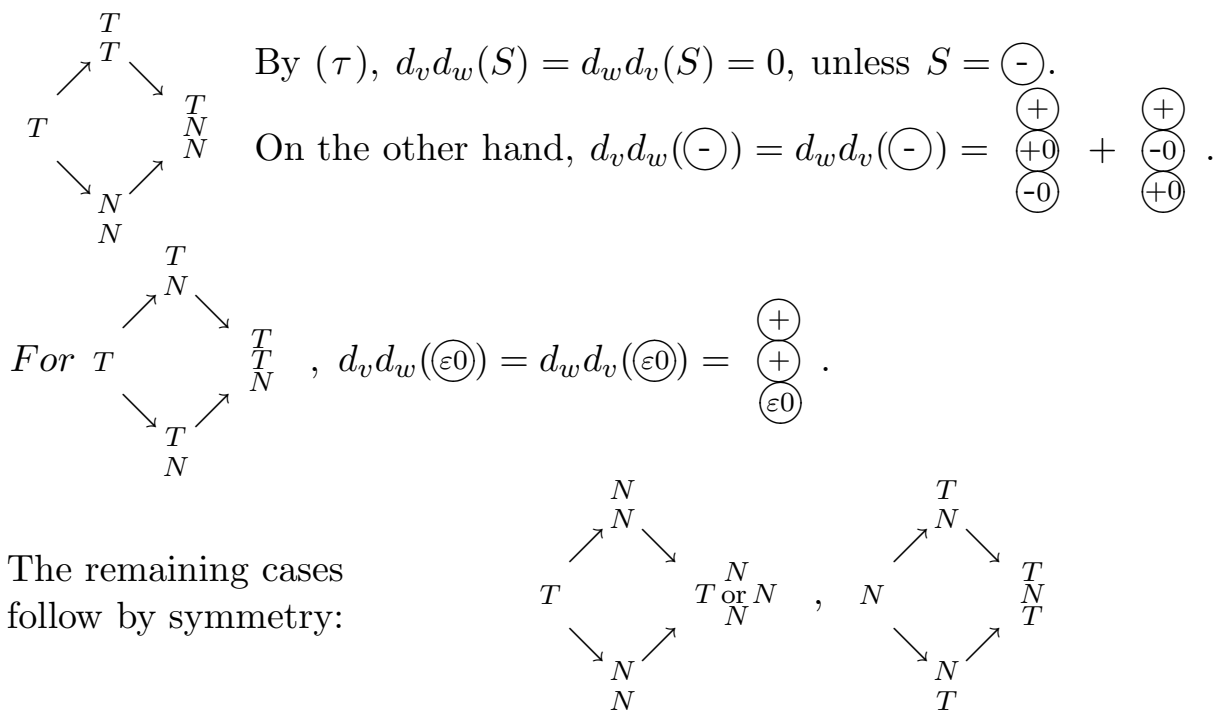

(2B) By $(\tau, \mathrm{NZ})$ we exclude the cases $(2 A, 2 C)=(N N, N N),(N N, T N)$, $(T N, N N)$. The remaining cases are:

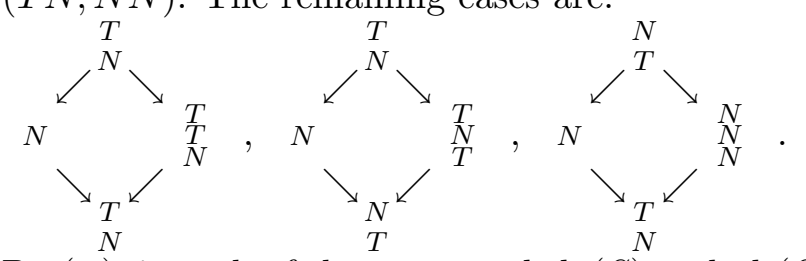

By $(\tau)$, in each of these cases, $d_{v} d_{w}(S)=d_{w} d_{v}(S)=0$, unless $S=\bigodot \varepsilon 0$.

For $S=\bigodot £$, we get $d_{v} d_{w}(S)=d_{w} d_{v}(S)=\stackrel{\oplus}{€ 0}$.

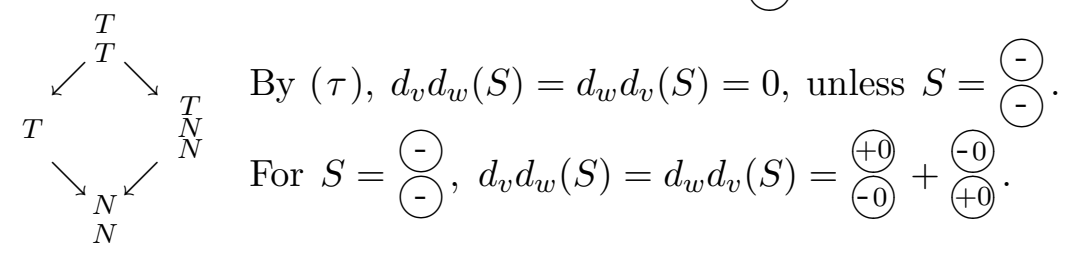

$(2 \mathrm{C})$ and (2D) follows from (2A), (2B) and Lemma 11.1 


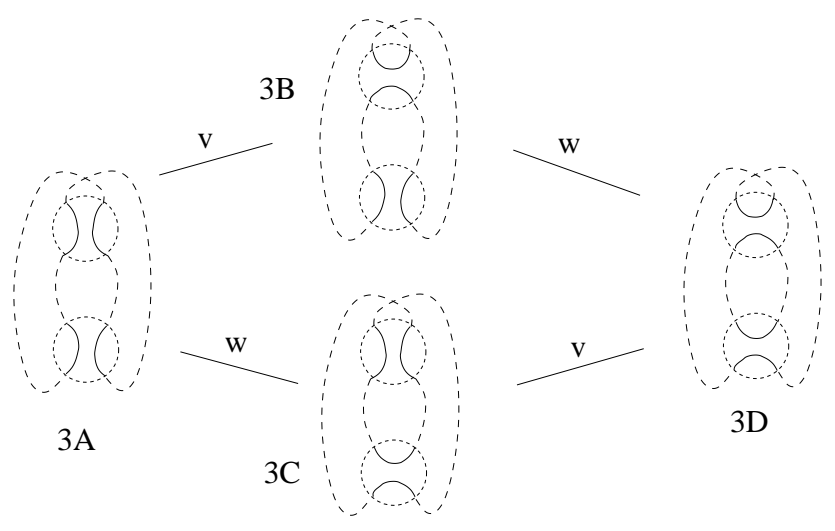

(3A) If $3 \mathrm{D}$ is $\mathrm{NN}$, then both sides of eq. (12) vanish by $(\tau)$. If $3 \mathrm{D} \in \mathrm{TT}$ then $3 \mathrm{~B}, 3 \mathrm{C}$ are $\mathrm{T}$ and (12) follows by $(\mathrm{S})$. Analogously, if $3 \mathrm{D}$ is $\mathrm{TN}$ then $3 \mathrm{~B}$, $3 \mathrm{C}$ are $\mathrm{N}$ and (12) follows by (S).

(3B) By $(\mathrm{NZ}, \tau)$ we assume that both $3 \mathrm{~B}$ and $3 \mathrm{C}$ are trivial and $S=-$. Hence $3 \mathrm{D}$ is either in $\mathrm{TT}$ or $\mathrm{NN}$. If $3 \mathrm{~A}$ is nontrivial then $d_{v} d_{w}(-)=$ $d_{w} d_{v}(\odot)=2 \odot$. Assume now that $3 \mathrm{~A}$ is trivial. Since $3 \mathrm{~B}, 3 \mathrm{C}$ are trivial as well, $F=\mathbb{R P}^{2}$. Since $d_{w} d_{v}(\odot)=0, d_{v} d_{w}(\odot)=2 \oplus$, (13) holds.

(3C) and (3D) follows from (3A), (3B) and Lemma 11.1.

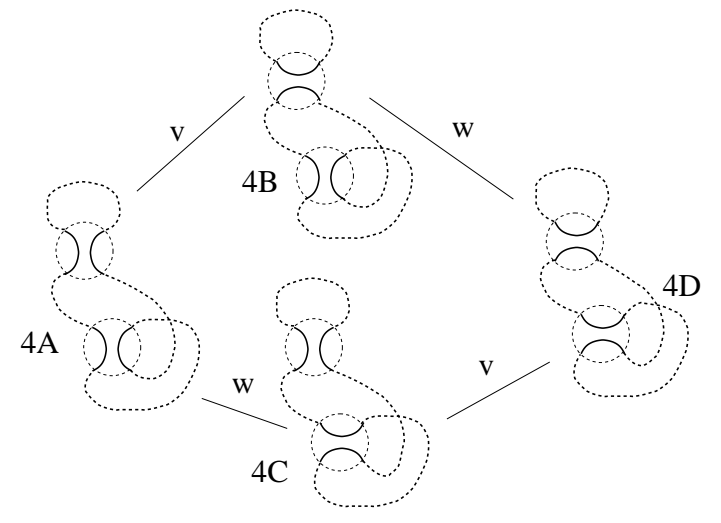

$(4 \mathrm{~A})$ By $(\tau, \mathrm{NZ}),(4 \mathrm{~A}, 4 \mathrm{D})=(\mathrm{T}, \mathrm{NT})$ or $(\mathrm{N}, \mathrm{TT})$. If $4 \mathrm{~A}=\mathrm{T}$, then $4 \mathrm{~B}=\mathrm{TT}$ or $\mathrm{NN}$. The following cases need to be considered:

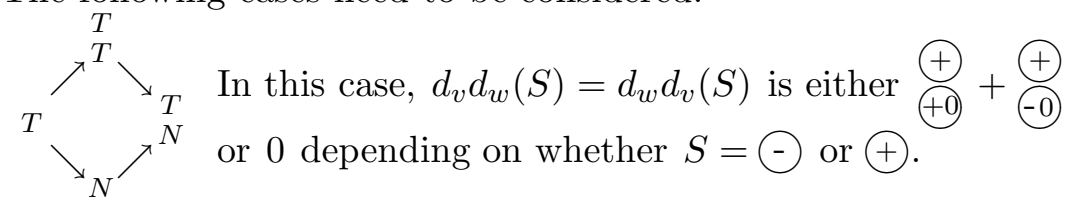




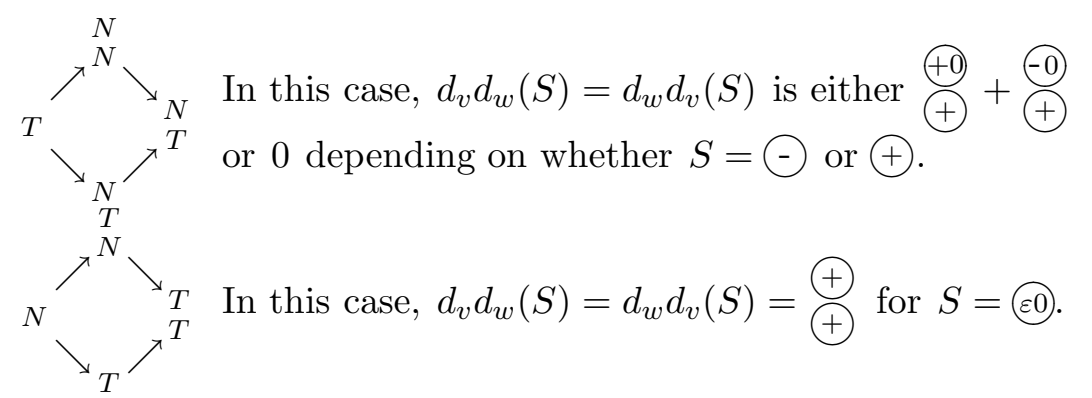

(4B) By $(\tau, \mathrm{NZ}),(4 \mathrm{~B}, 4 \mathrm{C})$ is either $(\mathrm{TT}, \mathrm{N})$ and $\mathrm{S}=--$ or $(\mathrm{TN}, \mathrm{T})$ and $\mathrm{S}=-(\varepsilon 0)$. In the first case, $4 \mathrm{~A}=\mathrm{T}$ and $4 \mathrm{D}={ }_{N}^{T}$. Hence $d_{v} d_{w}(\odot-)=d_{w} d_{v}(\odot \odot)=$ (+0) $+(-0$. Assume now that $4 \mathrm{~B}=\mathrm{TN}, 4 \mathrm{C}=\mathrm{T}$. If the top circle in $4 \mathrm{~B}$ is $\mathrm{T}$ then the top circle in $4 \mathrm{D}$ is also $\mathrm{T}$ and since $4 \mathrm{C}$ is $\mathrm{T}$, also the bottom circle of $4 \mathrm{D}$ is $\mathrm{T}$. Hence we have

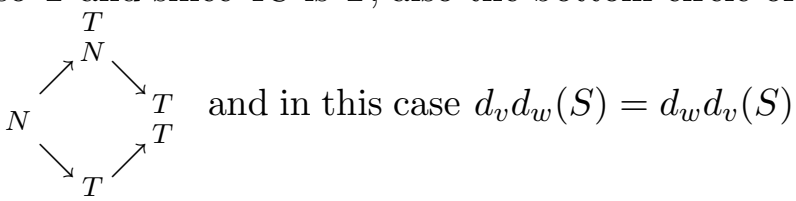
is $\oplus$ for $\mathrm{S}=\odot$, and 0 otherwise.

If the top circle in $4 \mathrm{~B}$ is $\mathrm{N}$ then the top circle in $4 \mathrm{D}$ is also $\mathrm{N}$ and since $4 \mathrm{C}$ is $\mathrm{T}$, the bottom circle of $4 \mathrm{D}$ is $\mathrm{N}$. Hence we have

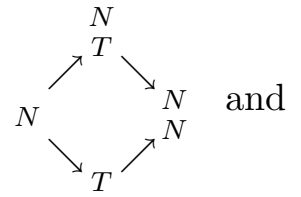
in this case $d_{v} d_{w}(S)=d_{w} d_{v}(S)$ is $\odot$ for $\mathrm{S}=\odot$, and 0 otherwise.

$(4 \mathrm{C})$ and $(4 \mathrm{D})$ follows from $(4 \mathrm{~A}),(4 \mathrm{~B})$ and Lemma 11.1.

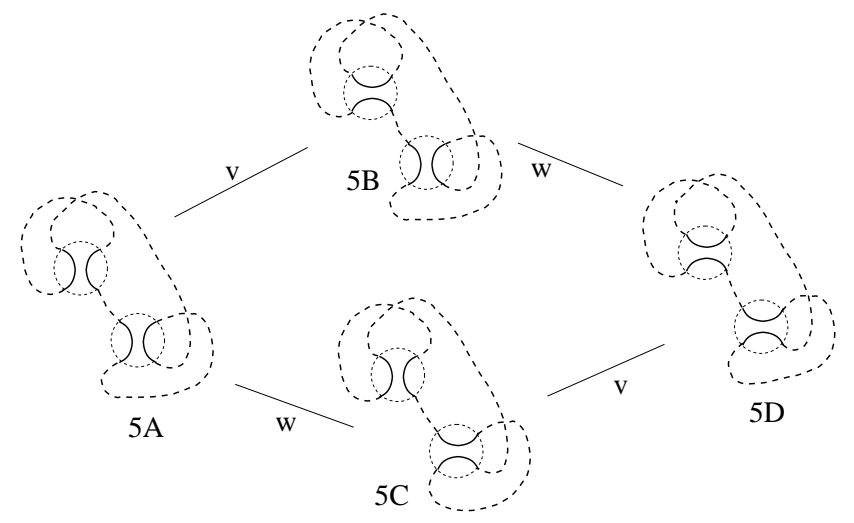

(5A) By $(\tau, \mathrm{NZ})$, we assume that both $5 \mathrm{~A}$ and $5 \mathrm{D}$ are trivial. Since for $(5 \mathrm{~B}, 5 \mathrm{C})=(\mathrm{T}, \mathrm{T})$ or $(\mathrm{N}, \mathrm{N}),(12)$ holds by $(\mathrm{S})$, assume that $(5 \mathrm{~B}, 5 \mathrm{C})=(\mathrm{T}, \mathrm{N})$. Since $(5 \mathrm{~A}, 5 \mathrm{~B})=(\mathrm{T}, \mathrm{T}), F=\mathbb{R P}^{2}$. In this case, $d_{w} d_{v}=0$, and $d_{v} d_{w}(S)=0$ $\bmod 2$ for all $S$. 
$(5 \mathrm{~B}),(5 \mathrm{C}),(5 \mathrm{D}) \quad$ The proof is identical.

This completes the proof of Theorem 5.1

\subsection{Reidemeister move I}

Let the link diagrams $D, D^{\prime}$ differ by a negative kink only, $D=\supset, D^{\prime}=\supset$ and let the map $\rho_{I}: C_{i j s}(つ) \rightarrow C_{i-1, j-3, s}(\supset)$ be given by $\stackrel{\varepsilon}{\varepsilon} \rightarrow \mathcal{\varepsilon}$, where $\epsilon= \pm, \pm 0$. One easily verifies that $\rho_{I}$ is a chain map. We are going to prove $\rho_{I}$ that induces an isomorphism on homology groups by induction on the number of crossings of $D$.

Assume first that $D$ has no crossings. It this situation one may assume that $D$ is a single loop. Let $s=D \in \mathcal{C}(F)$ if $D$ is non-bounding in $F$, and let $s=0$ otherwise. Since $C_{* * s^{\prime}}(D)=C_{* * s^{\prime}}\left(D^{\prime}\right)=0$, for $s^{\prime} \neq s$, it is enough to consider chain groups $C_{* * s}(D), C_{* * s}\left(D^{\prime}\right)$ only. Let $H_{i}(D)=\oplus_{j} H_{i j s}(D)$.

By direct calculation, $H_{i}(D)=0$ for $i \neq 0, H_{i}\left(D^{\prime}\right)=0$ for $i \neq-1$, and $H_{0}(D)$ and $H_{-1}\left(D^{\prime}\right)$ have bases $\{\oplus,-\odot\}$ and $\{\oplus \odot,-\odot \odot\}$ respectively, where $\epsilon$ is either 1 or 0 depending on whether $D$ is contractible or not. Since $\rho(\oplus)=$ $\left(\oplus-\rho(-\varepsilon)=-\odot \odot, \rho_{I}\right.$ induces an isomorphism $\rho_{I *}: H_{0}(D) \rightarrow H_{-1}\left(D^{\prime}\right)$, preserving the grading with respect to $j$ and $s$.

Now assume that $D$ is a link diagram with $c>0$ crossings and that $\rho_{I *}$ is an isomorphism on homology groups for all diagrams with at most $c-1$ crossings. Let $D^{\prime}$ be obtained from $D$ by adding a negative kink, as before. By inductive assumption, the long exact sequence of homology groups of $D$ for any crossing $p$ and the long exact sequence of homology groups of $D^{\prime}$ for the same $p$ are related by the maps $\rho_{I *}$ which, except for the central one, are isomorphisms by the inductive assumption:

$$
\begin{aligned}
& H_{i+2, j+2}\left(D_{0}\right) \stackrel{\partial}{\rightarrow} H_{i+2, j+4}\left(D_{\infty}\right) \stackrel{\alpha_{*}}{\rightarrow} H_{i+1, j+3}(D) \stackrel{\beta_{*}}{\rightarrow} H_{i, j+2}\left(D_{0}\right) \stackrel{\partial}{\rightarrow} H_{i, j+4}\left(D_{\infty}\right) \\
& \downarrow \rho_{I} \quad \downarrow \rho_{I} \quad \downarrow \rho_{I} \quad \downarrow \rho_{I} \quad \downarrow \rho_{I} \\
& H_{i+2, j+2}\left(D_{0}^{\prime}\right) \stackrel{\partial}{\rightarrow} H_{i+2, j+4}\left(D_{\infty}^{\prime}\right) \stackrel{\alpha_{*}}{\rightarrow} H_{i+1, j+3}\left(D^{\prime}\right) \stackrel{\beta_{*}}{\rightarrow} H_{i, j+2}\left(D_{0}^{\prime}\right) \stackrel{\partial}{\rightarrow} H_{i, j+4}\left(D_{\infty}^{\prime}\right) .
\end{aligned}
$$

(For brevity, we skip the index "s".)

Since for any state $S$ of $D, \rho_{I}(S)$ differs from $S$ by an additional - only, and that - does not pass trough any crossings of $D$, the above diagram commutes. Therefore, by Five lemma, $\rho_{I}: H_{* * *}(D) \rightarrow H_{* * *}\left(D^{\prime}\right)$ is an isomorphism and the proof of Theorem $6.2(2)$ is completed. 


\subsection{Invariance under Reidemeister move II}

Consider two link diagrams $\checkmark$ ' $\bigcirc$ ' related to each other by the second Reidemeister move and denoted by $D$ and $D^{\prime}$ respectively. These two diagrams differ by two vertices which will be denoted by $v$ and $w,{ }^{\mathrm{v}} /$. We assume that the vertices of $D^{\prime}$ are ordered such that $v$ is the first and that $w$ is the second vertex and that the vertices of $D$ are ordered by an ordering inherited from the ordering of vertices of $D^{\prime}$.

Let $f: C_{i j s}(\stackrel{\sim}{\sim}) \rightarrow C_{i j s}\left({ }^{\prime} \bigcirc^{\prime}\right)$ and $\left.g: C_{i j s}(\rangle\right) \rightarrow C_{i, j-2, s}\left({ }^{\prime} \bigcirc^{\prime}\right)$ be defined as follows:

$$
\underset{\delta}{\varepsilon} \stackrel{f}{\rightarrow} \underset{\delta}{\varepsilon}, \quad \varepsilon(\delta \stackrel{g}{\rightarrow} \varepsilon) \bar{O}(\delta .
$$

Let $\rho_{I I}: C_{i j s}(\stackrel{\sim}{\sim}) \rightarrow C_{i j s}\left({ }^{\prime}{ }^{\prime}\right)$ be given by

$$
\rho_{I I}(S)=f(S)+g(\gamma(S)) .
$$

(Recall that $\gamma: C_{i j s}(\stackrel{\sim}{\sim}) \rightarrow C_{i, j+2, s}()$ () was defined by (3]) in Section [7)

Theorem 11.3 (1) $\rho_{I I}$ is a chain map.

$$
\rho_{I I *}: H_{i j s}(\smile) \rightarrow H_{i j s}\left(\bigcirc^{\prime}\right) \text { is an isomorphism. }
$$

We start the proof with the following lemma whose proof is left to the reader.

Lemma 11.4 (1) For any enhanced state $\left.S \in C_{i, j+2, s}(\rangle\right), d g(S)=g d(S)+$ $(-1)^{m(S)+1} \imath(S)$, where $\imath(S) \in C_{i-2, j, s}\left(\bigcirc^{\prime}\right)$ is the enhanced state of the form $\smile$ < obtained by "deforming" the diagram of $S$. In other words, the labels of $S$ and $\imath(S)$ coincide under the obvious $1-1$ correspondence of circles of $S$ and $\imath(S)$.

(2) For any enhanced state $S \in C_{i j s}\left(\smile^{\smile}\right), d f(S)=f d(S)+(-1)^{m(S)} \sum S^{\prime}$, where the sum is taken over all enhanced states $S^{\prime} \in C_{i-2, j, s}\left(\bigcirc^{\prime}\right)$ of the form $\curvearrowleft$ (and such that the labels of all circles of $S$ and of $S^{\prime}$ coincide, except possibly the circles which pass through $\simeq$ in $S$ and through $\curvearrowleft\langle$ in $S$ '.

By Proposition $\left[7.2(4)\right.$, the sum of all states $S^{\prime}$ defined above equals to $\imath(\gamma(S))$. Hence

$$
d f(S)=f d(S)+(-1)^{m(S)} \imath \gamma(S) .
$$


If $\gamma(S)=\sum_{i} S_{i}$ then $m\left(S_{i}\right)=m(S)$ for all $i$, and therefore by Lemma 11.4(1)

$$
d g \gamma(S)=g d \gamma(S)+(-1)^{m(S)+1} \imath \gamma(S)
$$

and, by Proposition $[7.2(3)$,

$$
d g \gamma(S)=\operatorname{g\gamma d}(S)+(-1)^{m(S)+1} \imath \gamma(S) .
$$

By adding (15) and (16) we get the statement of Theorem 11.3(1).

The remaining part of this section is devoted to the proof of Theorem 11.3(2).

For any enhanced state $S$, let $m(S)$ denote the number of negative crossing markers in $S$ and let $\eta(S)=(-1)^{m(S)} S$.

Lemma $11.5 d \eta=-\eta d$

Proof It is enough to prove that $\hat{d}_{p} \eta(S)=-\eta \hat{d}_{p}(S)$ for each crossing $p$ in every state $S$. If the marker at $p$ is negative then both sides of the above equation vanish. Otherwise, $d_{p}(S)=\sum S^{\prime}$ and $m\left(S^{\prime}\right)=m(S)+1$ for every summand $S^{\prime}$ in $d_{p}(S)$.

Lemma 11.6 The following diagram commutes:

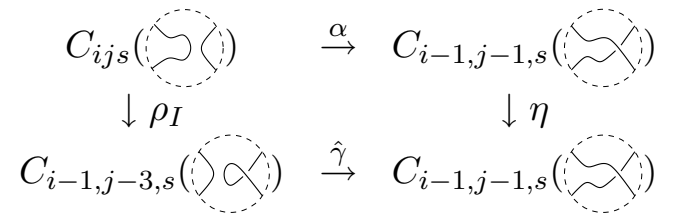

Proof By definition of $\alpha$ given in Section $7 \alpha(S)=\alpha_{0}(S)$ for any enhanced

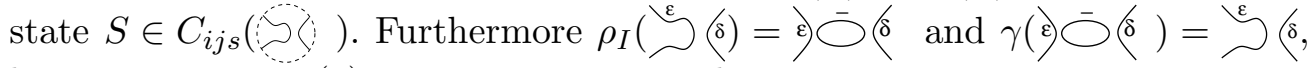
by Proposition $7.2(4)$. Hence $\gamma \rho_{I}=\alpha$, and

$$
\hat{\gamma} \rho_{I}(S)=(-1)^{m(S)+1} \gamma \rho_{I}(S)=(-1)^{m(S)+1} \alpha(S)=\eta \alpha(S) .
$$

Lemma 11.7 The following diagram commutes:

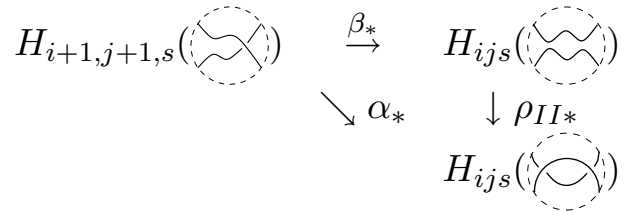


Proof Any $x \in C_{i+1, j+1, s}(-)$ decomposes into $x^{+}+x^{-}$where $x^{+}$(respectively: $x^{-}$) is a linear combination of states with positive (respectively: negative) marker at the crossing $w$. Assume that $x \in Z_{i+1, j+1, s}(\checkmark)$. Since $\beta\left(x^{-}\right)=0$, we need to show that

$$
\left(\rho_{I I} \beta-\alpha\right)\left(x^{+}+x^{-}\right)=f \beta\left(x^{+}\right)+g \gamma \beta\left(x^{+}\right)-\alpha\left(x^{+}\right)-\alpha\left(x^{-}\right)
$$

is a boundary cycle. Since the diagram

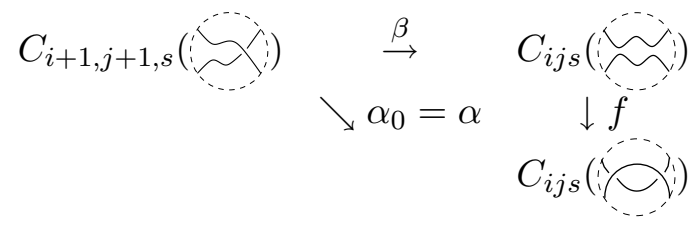

commutes for $x^{+}$, the right hand side of (17) equals $g \gamma \beta\left(x^{+}\right)-\alpha\left(x^{-}\right)$. Since

$$
\begin{gathered}
\gamma \beta=\hat{\gamma} \eta \beta=\bar{\alpha} \hat{d}_{w} \bar{\beta} \eta \beta=-\bar{\alpha} \eta \hat{d}_{w} \bar{\beta} \beta=-\bar{\alpha} \eta \hat{d}_{w}, \\
\left(\rho_{I I} \beta-\alpha\right)(x)=-g \bar{\alpha} \eta \hat{d}_{w}\left(x^{+}\right)-\alpha\left(x^{-}\right) .
\end{gathered}
$$

Since $x=x^{+}+x^{-} \in \operatorname{Ker} d$,

$$
\sum_{c} \hat{d}_{c}\left(x^{+}\right)+\sum_{c} \hat{d}_{c}\left(x^{-}\right)=0
$$

and by taking the states with the negative marker at $w$ into account only, we get $\hat{d}_{w}\left(x^{+}\right)+\sum_{c} \hat{d}_{c}\left(x^{-}\right)=0$. By substituting $-\sum_{c} \hat{d}_{c}\left(x^{-}\right)$for $d_{w}\left(x^{+}\right)$in (18) we get

$$
\left(\rho_{I I} \beta-\alpha\right)(x)=g \bar{\alpha} \eta\left(\sum_{c} \hat{d}_{c}\left(x^{-}\right)\right)-\alpha\left(x^{-}\right),
$$

and, since $g$ and $\bar{\alpha}$ commute with $\hat{d}_{c}$ for $c \neq v, w$, (cf. (4) ), and $\eta \hat{d}_{c}=-\hat{d}_{c} \eta$,

$$
\left(\rho_{I I} \beta-\alpha\right)(x)=-\sum_{c \neq v, w} \hat{d}_{c}\left(g \bar{\alpha} \eta\left(x^{-}\right)\right)-\alpha\left(x^{-}\right) .
$$

If $x^{-}=\sum_{i} a_{i} S_{i}$ and $S_{i}=\overbrace{}^{\varepsilon / \delta}$ then

$$
\left.g \bar{\alpha} \eta\left(S_{i}\right)=\varepsilon\right) \bar{\bigcirc}\left(\hat{\delta} \cdot(-1)^{m\left(S_{i}\right)} \in C_{i j s}\left(\bigcirc^{\prime}\right) .\right.
$$

Therefore

$$
d\left(g \bar{\alpha} \eta\left(S_{i}\right)\right)=\sum_{c \neq v, w} \hat{d}_{c}\left(g \bar{\alpha} \eta\left(S_{i}\right)\right)+\sum^{\varepsilon}\left(\delta \cdot(-1)^{m\left(S_{i}\right)}(-1)^{m\left(S_{i}\right)} .\right.
$$

Hence

$$
\left(\rho_{I I} \beta-\alpha\right)(x)=-d\left(g \bar{\alpha} \eta\left(x^{-}\right)\right) .
$$


Corollary 11.8 The following diagram commutes:

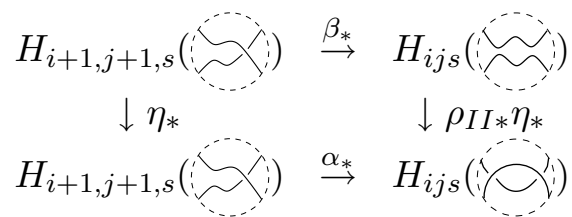

Proof Since $\eta$ skew-commutes with $\alpha, f$, and $g \gamma$, we have

$$
\alpha_{*} \eta_{*}=-\eta_{*} \alpha_{*}=-\eta_{*} \rho_{I I *} \beta_{*}=\rho_{I I *} \eta_{*} \beta_{*},
$$

by the previous lemma.

Lemma 11.9 The following diagram commutes:

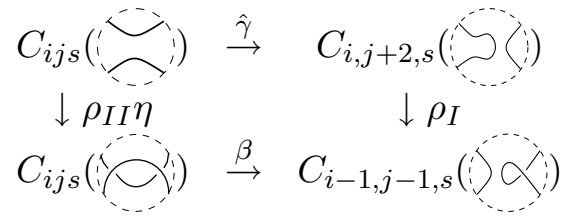

Proof Since $\beta f(S)=0$ for $S \in C_{i j s}\left(\begin{array}{l}) \\ )\end{array} \rho_{I I} \eta(S)=(-1)^{m(S)} \beta g \gamma(S)\right.$. Hence we need to prove that

$$
(-1)^{m(S)} \beta g \gamma(S)=(-1)^{m(S)} \rho_{I} \gamma(S) .
$$

Now the statement follows from the following commutative diagram:

$$
\begin{aligned}
& C_{i, j+2, s} \bigcirc(
\end{aligned}
$$

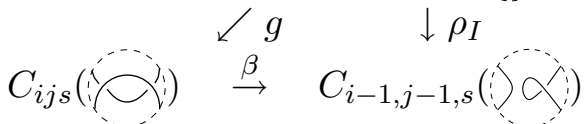

Consider the following two Viro's exact sequences

$$
\begin{aligned}
& H_{i+2, j+2}\left(\circlearrowleft() \stackrel{\alpha_{*}}{\rightarrow} H_{i+1, j+1}(\circlearrowleft) \stackrel{\beta_{*}}{\rightarrow} H_{i j}(D) \stackrel{\partial}{\rightarrow} H_{i, j+2}(\circlearrowleft \zeta) \stackrel{\alpha_{*}}{\rightarrow} H_{i-1, j+1}(\circlearrowleft)\right. \\
& \downarrow \rho_{I *} \quad \downarrow \eta_{*} \quad \downarrow \rho_{I I *} \eta_{*} \quad \downarrow \rho_{I *} \quad \downarrow \eta_{*}
\end{aligned}
$$

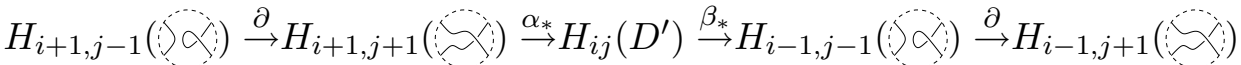

(The third index, $s$, is suppressed to shorten notation.)

By Lemma 11.6. Corollary 11.8, and Lemma11.9, the above diagram commutes. Hence, by Five Lemma, $\rho_{I I_{*}} \eta_{*}$ is an isomorphism. Since $\eta \eta=i d, \eta_{*}$ is an isomorphism, and hence $\rho_{I I *}$ is an isomorphism as well. This completes the proof of Theorem 11.3(2). 


\subsection{Invariance under Reidemeister move III}

In this section we prove the invariance of Khovanov homology under third Reidemeister moves. The proof is identical for homologies based on trivial and separating circles. Let $D, D^{\prime}$ be link diagrams related to each other by a third Reidemeister move shown below:

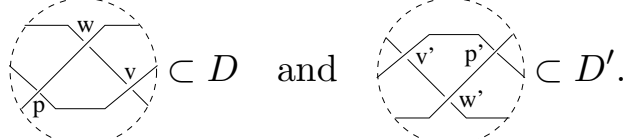

Assume that the vertices of $D$ and $D^{\prime}$ are ordered such that $p, v, w$ (respectively: $\left.p^{\prime}, v^{\prime}, w^{\prime}\right)$ are the first, the second, and the third among vertices of $D$ (respectively: of $D^{\prime}$ ) and that the orderings of the remaining vertices of $D$ and $D^{\prime}$ coincide. We denote by $D_{ \pm}$(respectively: by $D_{ \pm}^{\prime}$ ) the diagrams obtained from $D$ (respectively: $D^{\prime}$ ) by smoothing the crossing $p$ (respectively: $p^{\prime}$ ) with \pm marker.

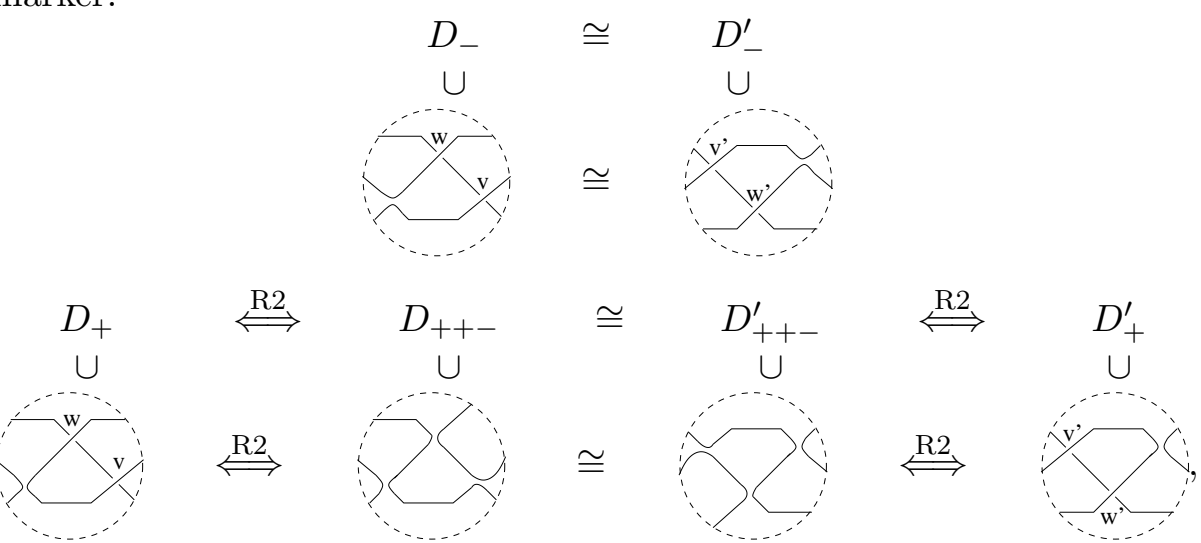

Let $\rho_{I I}: C_{* * *}\left(D_{++-}\right) \rightarrow C_{* * *}\left(D_{+}\right)$, and $\rho_{I I}^{\prime}: C_{* * *}\left(D_{++-}^{\prime}\right) \rightarrow C_{* * *}\left(D_{+}^{\prime}\right)$ be the isomorphisms defined in Section 11.4.

Since the diagrams $D_{-}$and $D_{-}^{\prime}$ are identical, except the crossing ordering change between vertices $v$ and $w$, their homology groups are isomorphic via the isomorphism $f: H_{i j s}\left(D_{-}\right) \rightarrow H_{i j s}\left(D_{-}^{\prime}\right)$ defined in the Section 11.1

Consider Viro's exact sequence:

$$
0 \rightarrow C_{i+1, j+1, s}\left(D_{-}\right) \stackrel{\alpha}{\rightarrow} C_{i j s}(D) \stackrel{\beta}{\rightarrow} C_{i-1, j-1, s}\left(D_{+}\right) \rightarrow 0 .
$$

Let $C_{i j s}^{\prime}\left(D_{+}\right)=\rho_{I I}\left(C_{i j s}\left(D_{++-}\right)\right)$and let $C_{i j s}^{\prime}(D)=\beta^{-1}\left(C_{i j s}^{\prime}\left(D_{+}\right)\right)$.

Proposition 11.10 The embeddings 
(1) $\quad C_{* * *}^{\prime}\left(D_{+}\right) \hookrightarrow C_{* * *}\left(D_{+}\right)$

(2) $C_{* * *}^{\prime}(D) \hookrightarrow C_{* * *}(D)$

induce isomorphisms on homology groups.

Proof (1) follows from Theorem 11.3(2).

(2) Since $\alpha\left(C_{i+1, j+1, s}\left(D_{-}\right)\right) \subset C_{i j s}^{\prime}(D)$, Viro's exact sequence restricts to

$$
0 \rightarrow C_{i+1, j+1, s}\left(D_{-}\right) \stackrel{\alpha}{\rightarrow} C_{i j s}^{\prime}(D) \stackrel{\beta}{\rightarrow} C_{i-1, j-1, s}^{\prime}\left(D_{+}\right) \rightarrow 0 .
$$

Therefore we have the following commuting diagram in which the vertical maps are induced by the natural embeddings:

$$
\begin{gathered}
H_{i+1, j-1}^{\prime}\left(D_{+}\right) \stackrel{\partial}{\rightarrow} H_{i+1, j+1}\left(D_{-}\right) \stackrel{\stackrel{\alpha_{*}}{\rightarrow}}{\rightarrow} H_{i j}^{\prime}(D) \stackrel{\stackrel{\beta_{*}}{\rightarrow}}{\rightarrow} H_{i-1, j-1}^{\prime}\left(D_{+}\right) \stackrel{\partial}{\rightarrow} H_{i-1, j+1}\left(D_{-}\right) \\
\downarrow \\
H_{i+1, j-1}\left(D_{+}\right) \stackrel{\partial}{\rightarrow} H_{i+1, j+1}\left(D_{-}\right) \stackrel{\alpha_{*}}{\rightarrow} H_{i j}(D) \stackrel{\beta_{*}^{\prime}}{\rightarrow} H_{i-1, j-1}\left(D_{+}\right) \stackrel{\partial}{\rightarrow} H_{i-1, j+1}\left(D_{-}\right) .
\end{gathered}
$$

Since the vertical morphisms $H_{* * *}\left(D_{-}\right) \rightarrow H_{* * *}\left(D_{-}\right)$are identity maps, the statement follows by Five Lemma.

Similarly, we define $C_{* * *}^{\prime}\left(D_{+}^{\prime}\right) \subset C_{* * *}\left(D_{+}^{\prime}\right)$ and $C_{* * *}^{\prime}\left(D^{\prime}\right) \subset C_{* * *}\left(D^{\prime}\right)$ and prove that these embeddings induce isomorphisms on homology groups.

Since $\rho_{I I}: C_{i j s}\left(D_{++-}\right) \rightarrow C_{i j s}^{\prime}\left(D_{+}\right)$is an isomorphism, the map $\rho=\rho_{I I}^{\prime} \rho_{I I}^{-1}$, $\rho: C_{* * *}^{\prime}\left(D_{+}\right) \rightarrow C_{* * *}^{\prime}\left(D_{+}^{\prime}\right)$, is well defined.

Proposition 11.11 The following diagram commutes:

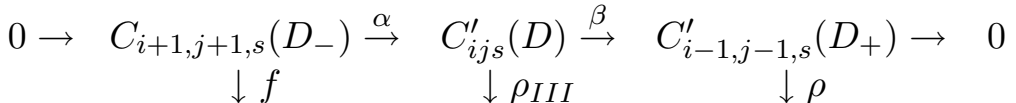

$$
\begin{aligned}
& 0 \rightarrow C_{i+1, j+1, s}\left(D_{-}^{\prime}\right) \stackrel{\alpha}{\rightarrow} C_{i j s}^{\prime}\left(D^{\prime}\right) \stackrel{\beta}{\rightarrow} C_{i-1, j-1, s}^{\prime}\left(D_{+}^{\prime}\right) \rightarrow 0,
\end{aligned}
$$

where $\rho_{I I I}=\bar{\beta} \rho \beta+\alpha f \bar{\alpha}$ and $\bar{\alpha}, \bar{\beta}$ are the maps defined in Section 7 .

Proof We have $\rho_{I I I} \alpha=(\bar{\beta} \rho \beta+\alpha f \bar{\alpha}) \alpha=\alpha f$ and $\beta \rho_{I I I}=\beta(\bar{\beta} \rho \beta-\alpha f \bar{\alpha})=$ $\rho \beta$.

Lemma 11.12 The following diagram commutes:

$$
\begin{array}{ccc}
C_{i+1, j-1, s}^{\prime}\left(D_{+}\right) & \stackrel{\hat{\gamma}}{\rightarrow} & C_{i+1, j+1, s}\left(D_{-}\right) \\
\downarrow \rho & & \downarrow f \\
C_{i+1, j-1, s}^{\prime}\left(D_{+}^{\prime}\right) & \stackrel{\hat{\gamma}}{\rightarrow} & C_{i+1, j+1, s}\left(D_{-}^{\prime}\right)
\end{array}
$$


Proof Since $\rho=\rho_{I I}^{\prime} \rho_{I I}^{-1}$, we need to show that

$$
f \hat{\gamma} \rho_{I I}(S)=\hat{\gamma} \rho_{I I}^{\prime}(S)
$$

for $S \in C_{* * *}\left(D_{++-}\right)$. If $S=\mathrm{a}^{\mathrm{b}} \mathrm{c} \in C_{i j s}\left(D_{++-}\right)$then

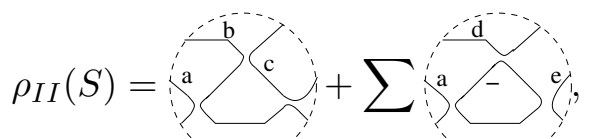

where the sum is over all possible labels $d, e$ for which the above states are in $C_{i j s}\left(D_{+}\right)$. Therefore, by (3) and Proposition [7.2(4),

$$
\hat{\gamma} \rho_{I I}(S)=(-1)^{m(S)+1} \sum \mathrm{g}_{\mathrm{g}}^{\mathrm{f}}+(-1)^{m(S)+1} \sum
$$

where $m(S)$ is the number of negative crossing markers of $S$, the second sum is as before, and the first sum is over all $f, g$ such that Since the states in the first sum have - and + markers at $v$ and $w$, respectively, and the states in the second sum have + and - markers at $v$ and $w$,

$$
f \hat{\gamma} \rho_{I I}(S)=\hat{\gamma} \rho_{I I}(S),
$$

by the definition of $f$.

Similarly considering $S$ as a state in $D_{++-}^{\prime}$, we get

$$
\hat{\gamma} \rho_{I I}^{\prime}(S)=(-1)^{m(S)+1} \sum+(-1)^{m(S)+1} \sum
$$

by (3) and Proposition 7.2(4).

Now (20), (21), and (22) imply (19).

Proposition 11.13 The map $\rho_{I I I}: C_{i j s}^{\prime}(D) \rightarrow C_{i j s}^{\prime}\left(D^{\prime}\right)$ defined in Proposition 11.11 is a chain map.

Proof If $S \in C_{i j s}^{\prime}(D)$ then

$$
\rho_{I I I} d(S)=\bar{\beta} \rho \beta d(S)+\alpha f \bar{\alpha} d(S) .
$$


Since $\beta$ and $\rho$ are chain maps and $\bar{\beta} d=\sum_{q \neq p} \hat{d}_{q} \bar{\beta}$, the first term on the right equals

$$
\bar{\beta} \rho \beta d(S)=\bar{\beta} d \rho \beta(S)=d \bar{\beta} \rho \beta(S)-\hat{d}_{p} \bar{\beta} \rho \beta(S) .
$$

We can write $S$ as $S_{+}+S_{-}$, where $S_{ \pm}$is composed of states of $D$ with \pm marker at the crossing $p$. Since $\bar{\alpha}$ commutes with $\hat{d}_{q}$ for $q \neq p$,

$$
\bar{\alpha} d(S)=\sum_{q \neq p} \hat{d}_{q} \bar{\alpha}(S)+\bar{\alpha} \hat{d}_{p}(S)=d \bar{\alpha}(S)+\bar{\alpha} \hat{d}_{p}\left(S_{+}\right) .
$$

Hence, for the second term on the right side of (23) we have

$$
\alpha f \bar{\alpha} d(S)=d \alpha f \bar{\alpha}(S)+\alpha f \bar{\alpha} \hat{d}_{p}\left(S_{+}\right) .
$$

Therefore, by (23), (24), and (25),

$$
\begin{gathered}
\rho_{I I I} d(S)=\left(d \bar{\beta} \rho \beta\left(S_{+}\right)-\hat{d}_{p} \bar{\beta} \rho \beta(S)\right)-\left(d \alpha f \bar{\alpha}(S)+\alpha f \bar{\alpha} \hat{d}_{p}\left(S_{+}\right)\right) \\
=d \rho_{I I I}(S)-X,
\end{gathered}
$$

where

$$
X=\hat{d}_{p} \bar{\beta} \rho \beta(S)-\alpha f \bar{\alpha} \hat{d}_{p}\left(S_{+}\right) .
$$

We are going to show that $X$ vanishes. Denote $\beta\left(S_{+}\right)$by $\tilde{S}_{+}$. Since $\hat{\gamma}=\bar{\alpha} \hat{d}_{p} \bar{\beta}$,

$$
X=\alpha \bar{\alpha} \hat{d}_{p} \bar{\beta} \rho\left(\tilde{S}_{+}\right)-\alpha f \bar{\alpha} \hat{d}_{p} \bar{\beta}\left(\tilde{S}_{+}\right)=\alpha \hat{\gamma} \rho\left(\tilde{S}_{+}\right)-\alpha f \hat{\gamma}\left(\tilde{S}_{+}\right) .
$$

Hence, $X$ vanishes by Lemma 11.12

Propositions 11.10 11.11 and 11.13 imply that the following diagram is exact and commutes:

$$
\begin{gathered}
H_{i+1, j-1, s}\left(D_{+}\right) \stackrel{\partial_{*}}{\rightarrow} H_{i+1, j+1, s}\left(D_{-}\right) \stackrel{\alpha_{*}}{\rightarrow} H_{i j s}(D) \stackrel{\beta_{*}}{\rightarrow} H_{i-1, j-1, s}\left(D_{+}\right) \stackrel{\partial_{*}}{\rightarrow} H_{i-1, j+1, s}\left(D_{-}\right) \\
\downarrow \rho_{*} \\
\downarrow f_{*}
\end{gathered}
$$

By Five Lemma $\rho_{I I I *}: H_{i j s}(D) \rightarrow H_{i j s}\left(D^{\prime}\right)$ is an isomorphism. Hence the proof of of Theorem 6.2(1) is completed. 


\section{References}

[AP] MM Asaeda, J H Przytycki, Khovanov homology: torsion and thickness, to appear in: "Advances in Topological Quantum Field Theory (Kananaskis Village, Canada 2001)", (John M. Bryden, editor), arXiv:math.GT/0402402

[APS] M M Asaeda, J H Przytycki, A S Sikora, A categorification of the skein module of tangles, arXiv:math.QA/0410238

[BN] D Bar-Natan, On Khovanov's categorification of the Jones polynomial, Algebr. Geom. Topol. 2 (2002) 337-270 MathReview

$[\mathrm{H}] \quad$ A Hatcher, Algebraic Topology, Cambridge University Press (2002) http://www . math. cornell.edu/ hatcher/AT/ATch3.pdf

[J] M Jacobsson, An invariant of link cobordisms from Khovanov's homology theory, Algebr. Geom. Topol. 4 (2004) 1211-1251

[K1] M Khovanov, A categorification of the Jones polynomial, Duke Math. J. 101 (2000) 359-426 MathReview

[K2] M Khovanov, Categorifications of the colored Jones polynomial, arXiv:math.QA/0302060

[K3] M Khovanov, An invariant of tangle cobordisms, arXiv:math.QA/0207264

[K4] M Khovanov, A functor-valued invariant of tangles, Algebr. Geom. Topol. 2 (2002) 665-741 MathReview

[Li] J B Listing, Vorstudien zur Topologie, Göttinger Studien (Abtheilung 1) 1 (1847) 811-875

[Pr] J H Przytycki, Fundamentals of Kauffman bracket skein modules, Kobe Math. J. 16 (1999) 45-66 MathReview

[PS] J H Przytycki, A S Sikora, On Skein Algebras and $S l_{2}(\mathbb{C})$-Character Varieties, Topology 39 (2000) 115-148 MathReview

[V] O Viro, Remarks on definition of Khovanov homology, Fund. Math. to appear, arXiv:math.GT/0202199

Dept of Mathematics, 14 MacLean Hall

University of Iowa, Iowa City, IA 52242, USA

Dept of Mathematics, Old Main Bldg, The George Washington University

1922 F St NW, Washington, DC 20052, USA

Dept of Mathematics, 244 Math Bldg, SUNY at Buffalo

Buffalo, NY 14260, USA and

Inst for Adv Study, School of Math, Princeton, NJ 08540, USA

Email: asaeda@math.uiowa.edu, przytyck@gwu.edu, asikora@buffalo.edu

Received: 23 September 2004 Revised: 6 December 2004 